\title{
PRODUCCIÓN CIENTÍFICA ESPAÑOLA EN TURISMO: UN ANÁLISIS DE AUTORÍA BASADO EN REVISTAS INTERNACIONALES CON ALTO IMPACTO Y VISIBILIDAD
}

\author{
Jesús Manuel López-Bonilla \\ Concepción Granados-Perea \\ Luis Miguel López-Bonilla \\ Universidad de Sevilla
}

\section{RESUMEN}

El presente estudio se ha centrado en el análisis de la producción científica con difusión internacional de los autores afiliados a instituciones españolas. La búsqueda se ha realizado a través de la base de datos de Scopus durante el período 2002-2013. Especialmente, se han analizado los trabajos publicados en revistas científicas que están incluidas en el Journal Citation Reports (JCR). Entre otros hallazgos, hay una gran concentración de autores que pertenecen a los ámbitos de la economía y la empresa. Las revistas científicas más utilizadas han sido Tourism Management y Tourism Economics.

Palabras clave: turismo; análisis de autoría; investigación; producción científica; Scopus; JCR; ranking.

\section{Spanish scientific production in tourism and hospitality: An analysis of authorship based on international journals with high impact and visibility}

\section{ABSTRACT}

This study has focused on the analysis of the international scientific output of scholars in Spain. The search was performed using the Scopus database from 2002 to 2013 . Especially,

Recibido: 28 de julio de 2016

Devuelto para su revisión: 20 de diciembre de 2016

Aceptado: 8 de febrero de 2017

Departamento de Administración de Empresas y Marketing. Universidad de Sevilla. Avda. Ramón y Cajal, 1. 41018 SEVILLA (España).E-mail: lopezbon@us.es, congraper@alum.us.es, luismi@us.es 
the papers published in scientific journals which are included in the Journal Citation Reports (JCR) were analysed. Among other findings, there is a significant concentration of authors from Economy and Business. The most used journals have been Tourism Management and Tourism Economics.

Keywords: tourism; authorship analysis; research; Scopus; scientific output; JCR; ranking.

\section{INTRODUCCIÓN}

La posición española en el contexto del turismo internacional es privilegiada desde hace muchos años, aunque la formación y la investigación en turismo se hayan incorporado más tarde en esta dinámica. En concreto, la formación universitaria en turismo se inicia con la creación de la Diplomatura de Turismo en 1996. La adaptación de España al Espacio Europeo de Educación Superior supone la aprobación de la Ley Orgánica 6/2001 de Universidades, que es modificada por la Ley Orgánica 4/2007, y que define la estructura de las enseñanzas universitarias en tres ciclos: Grado, Máster y Doctorado. Como indican Ceballos, Arias, Ruiz, Sanz y Vázquez (2010), la Unión Europea creó el Espacio Europeo de Educación Superior con idea de armonizar los sistemas universitarios de los países miembros. Por lo tanto, a partir de este desarrollo legislativo se ha conseguido que la formación universitaria en turismo pueda alcanzar los tres niveles de estudio de Grado, Máster y Doctorado.

Como consecuencia del escaso desarrollo de la formación universitaria en turismo, la investigación turística española empieza a consolidarse a partir del siglo XXI. Así, por ejemplo, España está situada en la quinta posición en el ranking mundial por número de documentos científicos que registra el Scimago Journal Rank en 2015 en el área de turismo, ocio y gestión de la hospitalidad (SCImago, 2017). Esta misma posición está corroborada por Park, Phillips, Canter y Abbott (2011) en su estudio bibliométrico realizado sobre las seis revistas principales en la investigación turística durante el período de 2000 a 2009.

Hay que precisar que todos los investigadores españoles pertenecen a una diversidad de áreas de conocimiento científico, sin ser ninguna de ellas un área específica de turismo al no existir en España. Muy probablemente, es escaso el número de investigadores españoles postdoctorales que han obtenido una titulación universitaria en turismo. Por ejemplo, el Ministerio de Educación, Cultura y Deporte (2017) publica entre sus últimos datos que el 0,4\% de las tesis doctorales leídas en el curso 2015-16 estaban adscritas a la especialidad de viajes, turismo y ocio. No obstante, los estudiantes de turismo ya pueden realizar estudios de doctorado en el ámbito turístico desde 2008. Además, en 2013, se ha constituido el primer Doctorado Interuniversitario en Turismo con el respaldo de la Universidad de Alicante, la Universidad de Málaga, la Universidad Rey Juan Carlos y la Universidad de Sevilla.

La tardía incorporación de las enseñanzas del turismo al catálogo de los estudios universitarios, además del escaso desarrollo de la investigación en ciencias sociales, ha contribuido a generar muchas dudas sobre la importancia de la investigación turística española. La mayoría de autores españoles han reflexionado sobre ello en varios estudios bibliométricos (Esteban, 2000; Vera e Ivars, 2001; López-Bonilla y LópezBonilla, 2004; Albacete-Sáez y Fuentes-Fuentes, 2010; Hernández, Campón y Folgado, 2011; Vargas, 2011a). Como ya indicaban López-Bonilla y López-Bonilla (2004) hace 
una década, la gran importancia que el sector turístico ha demostrado alcanzar en España no se ha correspondido con la atención prestada por la comunidad científica.

Recientemente, los investigadores españoles han mostrado interés en los análisis bibliométricos sobre la investigación en el ámbito del turismo. De este modo, se han realizado diversos estudios desde distintas perspectivas o disciplinas, tales como la geografía (v.gr., Antón, López, Marchena y Vera, 1996; Corral y San Eugenio, 2013; Corral y Cànoves, 2014), el marketing (v.gr., Bigné et al., 2008; López-Bonilla y López-Bonilla, 2008), la economía (v.gr., Bote, 1996; Figuerola, 2000; Aguiló, 2010) o la gestión de empresas (v.gr., Camisón y Monfort, 1996; Camisón et al., 1997; Sánchez y Marín, 2003; Ruiz, Chávez y Arias, 2006; Hernández, Campón y Folgado, 2011; Albacete, Fuentes y Haro-Domínguez, 2013). Pero también algunos estudios se han planteado con un carácter general o multidisciplinario (v.gr., Albacete-Sáez y Fuentes-Fuentes, 2010; Vargas, 2011a, 2011b; Moreno y Picazo, 2012; Corral y Cànoves, 2013; Corral-Marfil, Rodríguez, Vargas y Cànoves, 2015; López-Bonilla, Granados-Perea y López-Bonilla, 2017; Peláez-Verdet y Ferrera-Blasco, 2017). La mayoría de estos trabajos se ha basado en las publicaciones en revistas científicas, aunque hay otros que lo han hecho a través de las tesis doctorales (v.gr., Esteban, 2000; Ortega y Rodríguez, 2004; Agencia Valenciana de Turismo, 2006; López-Bonilla y López-Bonilla, 2008; Vargas, 2011b) o mediante las actas de congresos especializados en turismo (v.gr., Miralbel, Lamsfus, Gomis y González, 2012; Corral-Marfil, 2013; Corral y San Eugenio, 2013). No obstante, ninguno de estos trabajos se ha concentrado especialmente en el análisis de autorías.

Por todo ello, el objetivo principal del presente trabajo se ocupa de identificar a los autores prolíficos que están afiliados a instituciones españolas que han publicado sus trabajos en el ámbito turístico en revistas internacionales de alto impacto, considerando aquellas que están indexadas en las bases de datos más importantes a nivel mundial, como son Scopus y, especialmente, Web of Science (Journal Citation Reports). En concreto, se pretende analizar el número de artículos publicados por los autores prolíficos, su género, el número de coautoría, el número de citas recibidas, las revistas donde publican, así como las instituciones y áreas de conocimiento a las que pertenecen dichos autores. Como apuntan Zhao y Ritchie (2007), el análisis de autoría es un fenómeno mucho más reciente en la investigación turística que en otras disciplinas maduras. De este modo, se pretende analizar de manera descriptiva tanto la producción científica como la productividad de estos autores a través de algunos indicadores bibliométricos. Con ello se estima que se va a paliar en cierta medida la escasa investigación bibliométrica existente sobre los autores españoles que difunden sus estudios sobre turismo en el ámbito internacional. Asimismo, se contribuye a un mayor conocimiento de la investigación turística realizada en España por los autores más productivos con objeto de poder ser de utilidad para la comunidad científica, en general, así como para los investigadores más noveles que se aproximan a este ámbito de estudio.

\section{METODOLOGíA}

El presente estudio se ha planteado en torno a los autores que han publicado más trabajos sobre la investigación turística en el ámbito internacional y que están adscritos a instituciones españolas. Para ello, se ha elegido la base de datos Scopus, desde la que se han seleccionado inicialmente todos los autores y sus publicaciones. Algunos autores han 
manifestado ciertas ventajas de Scopus frente a Web of Science, basándose en su mayor cobertura de revistas (v.gr., Granda-Orive et al., 2013; Codina-Canet, Olmeda-Gómez y Perianes-Rodríguez, 2013). El período de estudio analizado abarca 12 años, desde 2002 hasta 2013, tratando de recoger el impulso inicial de la nueva Ley de universidades (Ley 6/2001, de 21 de diciembre de 2001), que emprende una reforma importante que va a estimular la investigación académica a través de los mayores incentivos de promoción profesional que conlleva el hecho de publicar en revistas con impacto internacional. Se ha aplicado un método de conteo por casos, que se ha basado en registrar el número de trabajos publicados por cada uno de los investigadores afiliados a instituciones españolas.

En un contexto internacional, los estudios que han tratado de identificar a los autores prolíficos en la investigación turística tienden a evaluar un número de revistas reducido, como ya han criticado algunos autores (v.gr., Hall, 2005; McKercher, 2008; Losekoot, Vergenis y Wood, 2001). La gran mayoría de estudios bibliométricos han consultado menos de diez revistas científicas. Muy pocos estudios han superado esta cifra, entre los que caben destacar los trabajos de Ryan (2005), que utiliza 16 revistas; McKercher (2007), que estudia 25 revistas; y Albacete, Fuentes y Haro-Domínguez (2013), que emplean 26 revistas. Asimismo, la gran mayoría de los trabajos revisados en la literatura han basado su análisis bibliométrico en revistas especializadas sobre turismo, salvo escasas excepciones (v.gr., Corral y Cánoves, 2013; Zhong, Wu y Morrison, 2015).

Por lo tanto, se ha creído conveniente analizar las revistas especializadas en turismo, pero también todas las demás publicaciones incluidas en Scopus. De este modo se ofrece una mayor cobertura a la escasez relativa de la producción científica española en turismo con difusión internacional hasta fechas recientes, así como en cuanto al número limitado de revistas especializadas sobre turismo que se han reconocido. En este sentido, se han detectado 52 revistas especializadas en las publicaciones de trabajos de ámbito turístico y que están registradas en Scopus en 2014. La casi totalidad de estas revistas están incluidas en la categoría de "Tourism, Leisure and Hospitality Management" de Scopus. Se han eliminado aquellos registros que se corresponden con Series de libros y no son propiamente revistas, como ocurre con Advances in Hospitality and Leisure y Tourism Social Sciences Series.

La selección de los trabajos publicados en revistas no especializadas en turismo se ha realizado a partir de la búsqueda de cuatro palabras claves que se identifican claramente con la investigación turística, como son las siguientes: "tourism" y "tourist", de carácter general y que abarcan la gran mayoría de trabajos publicados; y "hotel" y "travel agency", de carácter más específico al estar relacionadas con las principales empresas turísticas del sector. Esta búsqueda se ha realizado a través de los títulos de artículos, los resúmenes y las palabras claves (title, abstracts and keywords) de Scopus. Además, se ha realizado una última búsqueda complementaria a la anterior que se ha basado en buscar un amplio conjunto de palabras clave para encontrar nuevos trabajos de los autores prolíficos ya detectados, o bien, para hallar nuevos autores prolíficos, en su caso. Para ello, en concreto, se ha utilizado la totalidad de palabras clave que se recoge en el trabajo de Corral y Cànoves (2013), que suman la cincuentena de conceptos de búsqueda, y que se ha basado a su vez en el trabajo de González-Albo et al. (2009).

Además, en el proceso de búsqueda se han incluido exclusivamente aquellos trabajos que aparecen como publicaciones en revistas (identificadas en Scopus con el término de 
“journals”) y que hayan sido objeto de revisión científica, es decir, se han eliminado aquellos documentos que hayan sido considerados como editoriales, libros, capítulos de libros y reseñas de libros. En la selección de artículos se han considerado todos aquellos trabajos asignados con DOI y, al menos, publicados online por las revistas, aunque puede que algunos estén pendientes de su publicación definitiva en el proceso de búsqueda de este estudio. Asimismo, se ha realizado una exhaustiva labor de depuración de los trabajos publicados, sobre todo, en lo que respecta a las firmas de los autores, ya que muchos de ellos aparecen con distintas firmas y ha sido necesario reagruparlas para cada autor.

\section{RESULTADOS}

Las publicaciones sobre turismo en revistas con impacto internacional por parte de investigadores españoles han sido muy escasas durante el siglo $\mathrm{XX}$, como se ha comprobado en estudios previos (Albacete-Sáez y Fuentes-Fuentes, 2010; Albacete, Fuentes y Haro-Domínguez, 2013). A partir del año 2002 se inicia un verdadero impulso en la producción científica española que conlleva un crecimiento continuado hasta la actualidad. Siendo así, en el presente estudio se han analizado un total de 1864 publicaciones de autores pertenecientes a instituciones españolas en revistas científicas indexadas en Scopus y que han sido publicadas entre 2002 y 2013, de manera que el 74\% de ellas lo hicieron durante los últimos seis años de este período temporal. A continuación, se va a analizar, en primer lugar, los datos bibliométricos más generales para, posteriormente, pasar a observar aquellos datos más concretos del análisis de autoría.

En la tabla 1 se recogen todas las revistas científicas especializadas en turismo y que están indexadas en Scopus, en las que han publicado los autores españoles durante el período comprendido entre 2002 y 2013. Las diez primeras revistas especializadas pertenecen también a la base Web of Science (WoS), aunque destacan especialmente las dos primeras (Tourism Management y Tourism Economics). La gran mayoría de revistas tienen su origen en el Reino Unido, siendo 31 de las 45 revistas que aparecen. Además, ocho revistas son estadounidenses, mientras que el resto se circunscriben a varios países del arco mediterráneo (dos revistas croatas, una búlgara, una griega, una turca y una española).

En la tabla 2 se incluyen las revistas científicas internacionales, específicas y generales, en las que más publican los autores españoles. Hay 16 de las 30 revistas listadas que pertenecen netamente al ámbito turístico, liderando éstas las primeras posiciones. Así, Tourism Management destaca con 128 artículos, seguida de Tourism Economics, con 110 artículos. A mayor distancia aparece en tercer lugar Annals of Tourism Research, con 50 artículos. En cuarta posición se sitúa la primera revista española del listado y primera revista no específica de turismo, con 44 artículos publicados, que se corresponde con el Boletín de la Asociación de Geógrafos Españoles. Hay otras seis revistas españolas más entre las 30 revistas donde más publican los investigadores españoles en revistas indexadas en Scopus, siendo tres de ellas del área de Geografía (Estudios Geográficos, Document D’Analisi Geografica y, aunque más generalista de ciencias sociales, Scripta Nova), dos revistas del área de Economía y Empresa (Revista Galega de Economía e Investigaciones Europeas de Dirección y Economía de la Empresa) y una revista puramente del ámbito turístico (Cuadernos de Turismo). La nacionalidad más abundante de todas estas revistas es la de Reino Unido, con la mitad de ellas, destacando también tres revistas de origen estadounidense. 


\section{Tabla 1 \\ REVISTAS CIENTÍFICAS ESPECIALIZADAS EN INVESTIGACIÓN TURÍSTICA POR ORDEN DE PUBLICACIONES DE AUTORES ESPAÑOLES}

\begin{tabular}{|c|c|c|}
\hline REVISTA & $\begin{array}{l}\text { PAÍS DE } \\
\text { ORIGEN }\end{array}$ & $\begin{array}{c}\mathrm{N}^{\circ} \\
\text { ARTÍCULOS }\end{array}$ \\
\hline Tourism Management & Reino Unido & 128 \\
\hline Tourism Economics & Reino Unido & 110 \\
\hline Annals of Tourism Research & Reino Unido & 50 \\
\hline Journal of Travel Research & Reino Unido & 25 \\
\hline International Journal of Tourism Research & Reino Unido & 19 \\
\hline Journal of Travel and Tourism Marketing & Reino Unido & 17 \\
\hline Journal of Sustainable Tourism & Reino Unido & 16 \\
\hline International Journal of Contemporary Hospitality Management & Reino Unido & 15 \\
\hline Cornell Hospitality Quarterly & Estados Unidos & 14 \\
\hline Current Issues in Tourism & Reino Unido & 12 \\
\hline European Journal of Tourism Research & Bulgaria & 12 \\
\hline Tourismos & Grecia & 12 \\
\hline Anatolia: An International Journal of Tourism and Hospitality Research & Turquía & 11 \\
\hline Cuadernos de Turismo & España & 11 \\
\hline Tourism Geographics & Reino Unido & 10 \\
\hline Tourism & Croacia & 8 \\
\hline Tourism Analysis & Estados Unidos & 7 \\
\hline Tourism Management Perspectives & Estados Unidos & 6 \\
\hline Tourism and Hospitality Research & Reino Unido & 5 \\
\hline Journal of Hospitality, Leisure, Sports and Tourism Education & Reino Unido & 4 \\
\hline Journal of Hospitality and Tourism Technology & Reino Unido & 4 \\
\hline Journal of Policy Research in Tourism, Leisure and Events & Estados Unidos & 4 \\
\hline Journal of Tourism and Cultural Change & Reino Unido & 4 \\
\hline Journal of Vacation Marketing & Reino Unido & 4 \\
\hline e-Review of Tourism Research & Estados Unidos & 3 \\
\hline International Journal of Tourism Policy & Reino Unido & 3 \\
\hline Journal of Ecotourism & Reino Unido & 3 \\
\hline Journal of Hospitality and Tourism Research & Estados Unidos & 3 \\
\hline Journal of Hospitality Marketing and Management & Reino Unido & 3 \\
\hline Journal of Tourism History & Reino Unido & 3 \\
\hline Journal of Teaching in Travel and Tourism & Reino Unido & 2 \\
\hline Tourism and Hospitality Management & Croacia & 2 \\
\hline Tourist Studies & Reino Unido & 2 \\
\hline Visitor Studies & Reino Unido & 2 \\
\hline Asia Pacific Journal of Tourism Research & Reino Unido & 1 \\
\hline Journal of China Tourism Research & Reino Unido & 1 \\
\hline Journal of Human Resources in Hospitality and Tourism & Reino Unido & 1 \\
\hline Journal of Retail and Leisure Property & Reino Unido & 1 \\
\hline Journal of Quality Assurance in Hospitality and Tourism & Reino Unido & 1 \\
\hline
\end{tabular}




\begin{tabular}{|l|c|c|}
\hline Leisure Sciences & Estados Unidos & 1 \\
\hline Scandinavian Journal of Hospitality and Tourism & Reino Unido & 1 \\
\hline Tourism in Marine Environments & Estados Unidos & 1 \\
\hline Tourism Planning and Development & Reino Unido & 1 \\
\hline Tourism Review & Reino Unido & 1 \\
\hline Worldwide Hospitality and Tourism Themes & Reino Unido & 1 \\
\hline
\end{tabular}

Fuente: elaboración propia a partir de Scopus (2014).

Tabla 2

REVISTAS CIENTÍFICAS TOTALES EN LAS QUE PUBLICAN CON MAYOR FRECUENCIA LOS AUTORES ESPAÑOLES EN TURISMO

\begin{tabular}{|c|c|c|}
\hline REVISTA & $\begin{array}{l}\text { PAIS DE } \\
\text { ORIGEN }\end{array}$ & $\mathrm{N}^{\mathrm{o}}$ ART. \\
\hline Tourism Management & Reino Unido & 128 \\
\hline Tourism Economics & Reino Unido & 110 \\
\hline Annals of Tourism Research & Reino Unido & 50 \\
\hline Boletín de la Asociación de Geógrafos Españoles & España & 44 \\
\hline Service Industries Journal & Reino Unido & 34 \\
\hline International Journal of Hospitality Management & Reino Unido & 29 \\
\hline Journal of Coastal Research & Estados Unidos & 25 \\
\hline Journal of Travel Research & Reino Unido & 24 \\
\hline Estudios Geográficos & España & 21 \\
\hline Witt Transaction on Ecology and the Environment & Reino Unido & 21 \\
\hline International Journal of Tourism Research & Reino Unido & 19 \\
\hline Document D'Analisi Geografica & España & 18 \\
\hline Journal of Travel and Tourism Marketing & Reino Unido & 17 \\
\hline Journal of Sustainable Tourism & Reino Unido & 16 \\
\hline Interntional Journal of Contemporary Hospitality Management & Reino Unido & 15 \\
\hline Revista Galega de Economía & España & 15 \\
\hline Cornell Hospitality Quarterly & Estados Unidos & 14 \\
\hline Desalination & Holanda & 13 \\
\hline Current Issues in Tourism & Reino Unido & 12 \\
\hline European Journal of Tourism Research & Bulgaria & 12 \\
\hline Investigaciones Europeas de Dirección y Economía de la Empresa & España & 12 \\
\hline Ocean and Coastal Management & Reino Unido & 12 \\
\hline Tourismos & Grecia & 12 \\
\hline Anatolia: An International Journal of Tourism and Hospitality Research & Turquía & 11 \\
\hline Cuadernos de Turismo & España & 11 \\
\hline Environmental Engineering and Management Journal & Rumanía & 11 \\
\hline Expert Systems with Applications & Reino Unido & 11 \\
\hline Scripta Nova & España & 11 \\
\hline Environmental Management & Estados Unidos & 10 \\
\hline Tourism Geographies & Reino Unido & 10 \\
\hline
\end{tabular}

Fuente: elaboración propia a partir de Scopus (2014). 
A continuación, en la tabla 3 se detallan las instituciones españolas de las que proceden los autores españoles en investigación turística. Se puede observar que se trata de 25 universidades, cuyo ranking está liderado por la Universidad de Islas Baleares, con 139 artículos publicados, aunque le sigue muy de cerca la Universidad de Alicante, con 128 artículos. Se puede apreciar también que existe una clara concentración de publicaciones en las zonas geográficas españolas que poseen una mayor actividad turística, como son Baleares, Canarias, Comunidad Valenciana, Cataluña y Andalucía.

Tabla 3

INSTITUCIONES DE PROCEDENCIA DE LOS AUTORES ESPAÑOLES EN INVESTIGACIÓN TURÍSTICA

\begin{tabular}{|c|c|c|}
\hline UNIVERSIDADES & CC.AA. & No ARTÍCULOS \\
\hline Universidad de Islas Baleares & Islas Baleares & 139 \\
\hline Universidad de Alicante & Comunidad Valenciana & 128 \\
\hline Universidad de Valencia & Comunidad Valenciana & 92 \\
\hline Universidad de Las Palmas de Gran Canaria & Islas Canarias & 78 \\
\hline Universidad de Málaga & Andalucía & 76 \\
\hline Universidad Autónoma de Barcelona & Cataluña & 75 \\
\hline Universidad de Barcelona & Cataluña & 67 \\
\hline Universidad Autónoma de Madrid & Comunidad de Madrid & 65 \\
\hline Universidad de Sevilla & Andalucía & 59 \\
\hline Universidad Complutense & Comunidad de Madrid & 58 \\
\hline Universidad de Castilla-La Mancha & C. Castilla-La Mancha & 52 \\
\hline Universidad Politécnica de Valencia & Comunidad Valenciana & 48 \\
\hline Universidad de Girona & Cataluña & 45 \\
\hline Universidad de Granada & Andalucía & 45 \\
\hline Universidad de Vigo & Galicia & 45 \\
\hline Universidad de La Laguna & Islas Canarias & 41 \\
\hline Universidad de Cantabria & Cantabria & 37 \\
\hline UNED & Comunidad de Madrid & 34 \\
\hline Universidad de Santiago de Compostela & Galicia & 33 \\
\hline Universidad de Murcia & Murcia & 32 \\
\hline Universidad Jaume I & Comunidad Valenciana & 29 \\
\hline Universidad Pablo de Olavide & Andalucía & 28 \\
\hline Universidad Roviri i Virgili & Cataluña & 27 \\
\hline Universidad de Alcalá de Henares & Comunidad de Madrid & 26 \\
\hline Universidad de Almería & Andalucía & 26 \\
\hline
\end{tabular}

Fuente: elaboración propia a partir de Scopus (2014). 
En cuanto a los autores españoles más prolíficos que han publicado sus trabajos en Scopus en estos 12 años se encuentran los diez siguientes: J.L. Nicolau (Universidad de Alicante), con 27 artículos; E. Claver-Cortés (Universidad de Alicante), con 26 artículos; J. Rosselló (Universidad de Islas Baleares), con 24 artículos; I. Gil Saura (Universidad de Valencia) y D.M. Frías Jamilena (Universidad de Granada), con 21 artículos cada autora; J.F. Molina-Azorín (Universidad de Alicante) y J. Pereira-Moliner (Universidad de Alicante), con 20 artículos cada autor; J. Alegre (Universidad de Islas Baleares) y M.M. Alonso-Almeida (Universidad Autónoma de Madrid), con 19 artículos en cada caso; y M.J. Such Devesa (Universidad de Alcalá de Henares), con 16 artículos. Entre ellos se puede distinguir un claro predominio de autores de la Universidad de Alicante, con cuatro de ellos, seguido de la Universidad de Islas Baleares, con dos autores.

El presente trabajo no desea limitarse a un análisis meramente cuantitativo en el que se recojan todos los trabajos que han publicado los autores prolíficos españoles. De alguna manera, se pretende realizar también un análisis cualitativo para establecer un ranking de carácter mixto que considere tanto la producción como la productividad científica de los autores. Para ello, se valora el número de trabajos publicados en el Journal Citation Reports (JCR) como un indicador principal que aporta un mayor valor añadido a las publicaciones de los autores. En la tabla 4 se detallan los autores prolíficos, las instituciones de procedencia y sus áreas de conocimiento, así como los porcentajes de publicaciones JCR respecto al total de publicaciones en Scopus.

Tabla 4

\section{AUTORES PROLÍFICOS ESPAÑOLES EN INVESTIGACIÓN TURÍSTICA CON PUBLICACIONES JCR}

\begin{tabular}{|c|c|c|c|c|c|c|}
\hline \multirow{2}{*}{ Ord. } & \multirow{2}{*}{ Autores } & \multicolumn{2}{|c|}{ Afiliación de autores } & \multirow{2}{*}{$\begin{array}{c}\mathrm{N}^{\circ} \text { trabajos } \\
\text { JCR }\end{array}$} & \multirow{2}{*}{$\begin{array}{c}\mathrm{N}^{\circ} \text { trabajos } \\
\text { Scopus }\end{array}$} & \multirow{2}{*}{$\begin{array}{c}\% \\
\text { JCR }\end{array}$} \\
\hline & & Institución & Área de conocimiento & & & \\
\hline 1 & J.L. Nicolau & Universidad de Alicante & $\begin{array}{c}\text { Comercialización e } \\
\text { Investigación Mercados }\end{array}$ & 26 & 27 & $96,3 \%$ \\
\hline 2 & J. Rosselló & $\begin{array}{c}\text { Universidad Islas } \\
\text { Baleares } \\
\end{array}$ & Economía Aplicada & 20 & 24 & $83,3 \%$ \\
\hline 3 & D.M. Frías Jamilena & Universidad de Granada & $\begin{array}{c}\text { Comercialización e } \\
\text { Investigación Mercados }\end{array}$ & 17 & 21 & $81 \%$ \\
\hline 4 & E. Claver-Cortés & Universidad de Alicante & $\begin{array}{c}\text { Organización de } \\
\text { Empresas }\end{array}$ & 16 & 26 & $61,5 \%$ \\
\hline 5 & M.M. Alonso-Almeida & $\begin{array}{l}\text { Univ. Autónoma de } \\
\text { Madrid }\end{array}$ & $\begin{array}{l}\text { Organización de } \\
\text { Empresas }\end{array}$ & 16 & 19 & $84,2 \%$ \\
\hline 6 & J.F. Molina-Azorín & Universidad de Alicante & $\begin{array}{l}\text { Organización de } \\
\text { Empresas }\end{array}$ & 15 & 20 & $75 \%$ \\
\hline 7 & J. Alegre & $\begin{array}{c}\text { Universidad Islas } \\
\text { Baleares }\end{array}$ & Economía Aplicada & 13 & 19 & $68,4 \%$ \\
\hline 8 & J. Pereira-Moliner & Universidad de Alicante & $\begin{array}{l}\text { Organización de } \\
\text { Empresas }\end{array}$ & 12 & 20 & $60 \%$ \\
\hline 9 & M.A. Rodríguez-Molina & Universidad de Granada & $\begin{array}{c}\text { Comercialización e } \\
\text { Investigación Mercados }\end{array}$ & 12 & 15 & $80 \%$ \\
\hline 10 & J. Rey-Maquieira & $\begin{array}{c}\text { Universidad Islas } \\
\text { Baleares }\end{array}$ & Economía Aplicada & 11 & 15 & $73,3 \%$ \\
\hline
\end{tabular}




\begin{tabular}{|c|c|c|c|c|c|c|}
\hline 11 & B. Plaza & $\begin{array}{c}\text { Universidad del País } \\
\text { Vasco }\end{array}$ & Economía Aplicada & 9 & 9 & $100 \%$ \\
\hline 11 & J.A. Jiménez & $\begin{array}{c}\text { Universidad Politécnica } \\
\text { de Cataluña }\end{array}$ & Ingeniería Hidráulica & 9 & 9 & $100 \%$ \\
\hline 13 & A.I. Polo-Peña & Universidad de Granada & $\begin{array}{c}\text { Comercialización e } \\
\text { Investigación Mercados }\end{array}$ & 8 & 11 & $72,7 \%$ \\
\hline 14 & I. Rodríguez del Bosque & $\begin{array}{l}\text { Universidad de } \\
\text { Cantabria }\end{array}$ & $\begin{array}{c}\text { Comercialización e } \\
\text { Investigación Mercados }\end{array}$ & 8 & 10 & $80 \%$ \\
\hline 15 & J.A. Castañeda-García & Universidad de Granada & $\begin{array}{c}\text { Comercialización e } \\
\text { Investigación Mercados }\end{array}$ & 8 & 9 & $88,9 \%$ \\
\hline 15 & J.M. López-Bonilla & Universidad de Sevilla & $\begin{array}{c}\text { Comercialización e } \\
\text { Investigación Mercados }\end{array}$ & 8 & 9 & $88,9 \%$ \\
\hline 15 & L.M. López-Bonilla & Universidad de Sevilla & $\begin{array}{c}\text { Comercialización e } \\
\text { Investigación Mercados }\end{array}$ & 8 & 9 & $88,9 \%$ \\
\hline 18 & J.I. Castillo-Manzano & Universidad de Sevilla & Economía Aplicada & 8 & 8 & $100 \%$ \\
\hline 18 & H. San Martín & $\begin{array}{c}\text { Universidad de } \\
\text { Cantabria }\end{array}$ & $\begin{array}{c}\text { Comercialización e } \\
\text { Investigación Mercados }\end{array}$ & 8 & 8 & $100 \%$ \\
\hline 20 & A. Riera-Font & $\begin{array}{c}\text { Universidad Islas } \\
\text { Baleares }\end{array}$ & Economía Aplicada & 7 & 11 & $63,4 \%$ \\
\hline 21 & E. Bigné-Alcañiz & $\begin{array}{l}\text { Universidad de } \\
\text { Valencia }\end{array}$ & $\begin{array}{c}\text { Comercialización e } \\
\text { Investigación Mercados }\end{array}$ & 7 & 9 & $77,8 \%$ \\
\hline 21 & J.I. Pulido-Fernández & Universidad de Jaén & Economía Aplicada & 7 & 9 & $77,8 \%$ \\
\hline 21 & V. Ramos & $\begin{array}{c}\text { Universidad Islas } \\
\text { Baleares }\end{array}$ & Economía Aplicada & 7 & 9 & $77,8 \%$ \\
\hline 21 & J.M. Peiró & $\begin{array}{l}\text { Universidad de } \\
\text { Valencia }\end{array}$ & Psicología Social & 7 & 9 & $77,8 \%$ \\
\hline 25 & D.R. Medina-Muñoz & $\begin{array}{c}\text { Universidad de Las } \\
\text { Palmas de Gran Canaria }\end{array}$ & $\begin{array}{l}\text { Organización de } \\
\text { Empresas }\end{array}$ & 7 & 8 & $87,5 \%$ \\
\hline 25 & R.D. Medina-Muñoz & \begin{tabular}{|c|} 
Universidad de Las \\
Palmas de Gran Canaria
\end{tabular} & $\begin{array}{l}\text { Organización de } \\
\text { Empresas }\end{array}$ & 7 & 8 & $87,5 \%$ \\
\hline 25 & F. Orfila-Sintes & $\begin{array}{c}\text { Universidad Islas } \\
\text { Baleares }\end{array}$ & $\begin{array}{l}\text { Organización de } \\
\text { Empresas }\end{array}$ & 7 & 8 & $87,5 \%$ \\
\hline 25 & R. Rigal-I-Torrel & Universidad de Girona & $\begin{array}{c}\text { Fundamentos Análisis } \\
\text { Económico }\end{array}$ & 7 & 8 & $87,5 \%$ \\
\hline 29 & V. Martínez-Tur & $\begin{array}{l}\text { Universidad de } \\
\text { Valencia }\end{array}$ & Psicología Social & 7 & 7 & $100 \%$ \\
\hline 29 & E. Ruiz-Ballesteros & Univ. Pablo de Olavide & Antropología & 7 & 7 & $100 \%$ \\
\hline 29 & M. Santana-Gallego & $\begin{array}{c}\text { Universidad Islas } \\
\text { Baleares }\end{array}$ & Economía Aplicada & 7 & 7 & $100 \%$ \\
\hline 32 & M.J. Such Devesa & $\begin{array}{c}\text { Univ. de Alcalá de } \\
\text { Henares }\end{array}$ & Economía Aplicada & 6 & 16 & $37,5 \%$ \\
\hline 33 & L.A. Gil-Alaña & Universidad de Navarra & $\begin{array}{c}\text { Métodos Cuantitativos } \\
\text { para Economía y } \\
\text { Empresa }\end{array}$ & 6 & 12 & $50 \%$ \\
\hline 34 & A. Molina Collado & $\begin{array}{l}\text { Univ. de Castilla-La } \\
\text { Mancha }\end{array}$ & $\begin{array}{c}\text { Comercialización e } \\
\text { Investigación Mercados }\end{array}$ & 6 & 10 & $60 \%$ \\
\hline 34 & J.J. Tari Guillo & Universidad de Alicante & $\begin{array}{l}\text { Organización de } \\
\text { Empresas }\end{array}$ & 6 & 10 & $60 \%$ \\
\hline 36 & E. Aguiló & \begin{tabular}{|l} 
Universidad Islas \\
Baleares
\end{tabular} & Economía Aplicada & 6 & 9 & $66,7 \%$ \\
\hline 36 & C.J. León González & $\begin{array}{c}\text { Universidad de Las } \\
\text { Palmas de Gran Canaria }\end{array}$ & Economía Aplicada & 6 & 9 & $66,7 \%$ \\
\hline 38 & L. Andreu & $\begin{array}{l}\text { Universidad de } \\
\text { Valencia }\end{array}$ & $\begin{array}{c}\text { Comercialización e } \\
\text { Investigación Mercados }\end{array}$ & 6 & 8 & $75 \%$ \\
\hline
\end{tabular}




\begin{tabular}{|c|c|c|c|c|c|c|}
\hline 38 & J.B. Garau-Vadell & $\begin{array}{c}\text { Universidad Islas } \\
\text { Baleares } \\
\end{array}$ & $\begin{array}{c}\text { Comercialización e } \\
\text { Investigación Mercados }\end{array}$ & 6 & 8 & $75 \%$ \\
\hline 40 & G. Anfuso & Universidad de Cádiz & Geología & 6 & 7 & $85,7 \%$ \\
\hline 40 & M. Sánchez-Rivero & $\begin{array}{l}\text { Universidad de } \\
\text { Extremadura }\end{array}$ & $\begin{array}{c}\text { Métodos Cuantitativos } \\
\text { para Economía y } \\
\text { Empresa }\end{array}$ & 6 & 7 & $85,7 \%$ \\
\hline 40 & J. Uche-Marcuello & $\begin{array}{c}\text { Universidad de } \\
\text { Zaragoza }\end{array}$ & $\begin{array}{c}\text { Máquinas y Motores } \\
\text { Térmicos }\end{array}$ & 6 & 7 & $85,7 \%$ \\
\hline 43 & A. García-Pozo & Universidad de Málaga & Economía Aplicada & 6 & 6 & $100 \%$ \\
\hline 43 & J. Ramos & $\begin{array}{c}\text { Universidad de } \\
\text { Valencia }\end{array}$ & Psicología Social & 6 & 6 & $100 \%$ \\
\hline 43 & J.M. Rodríguez Antón & $\begin{array}{l}\text { Univ. Autónoma de } \\
\text { Madrid }\end{array}$ & $\begin{array}{c}\text { Organización de } \\
\text { Empresas }\end{array}$ & 6 & 6 & $100 \%$ \\
\hline 43 & J.L. Sánchez-Ollero & Universidad de Málaga & Economía Aplicada & 6 & 6 & $100 \%$ \\
\hline
\end{tabular}

Fuente: elaboración propia a partir de Scopus (2014) y JCR (2014).

En la tabla 5 se puede contemplar también que existe una gran concentración de las áreas de conocimiento en las que están adscritos los autores prolíficos españoles. De este modo, destacan especialmente las áreas de Economía Aplicada y de Marketing, con 14 y 13 autores en cada una de ellas, respectivamente. El área de Economía Aplicada tiene un mayor número de publicaciones, alcanzando a 124 artículos en Scopus, de los cuales 94 se publicaron también en JCR. En cambio el área de Marketing consigue una mayor eficacia de sus artículos al publicar el 81,6\% de ellos en revistas JCR. A mayor distancia, en tercera posición se sitúa el área de Organización de Empresas, con nueve autores y 64 artículos publicados en Scopus, siendo 47 de ellos publicados también en JCR. Hay otras áreas de conocimiento que aparecen de manera mucho más limitada tanto en número de autores como en número de publicaciones.

\section{TABLA 5 \\ ÁREAS DE CONOCIMIENTO PRINCIPALES DE AUTORES PROLÍFICOS ESPAÑOLES CON PUBLICACIONES JCR}

\begin{tabular}{|l|c|c|c|c|}
\hline ÁREAS DE CONOCIMIENTO & $\begin{array}{c}\mathrm{N}^{\circ} \\
\text { Autores }\end{array}$ & $\begin{array}{c}\mathrm{N}^{\mathrm{o}} \text { Trabajos } \\
\text { Scopus }\end{array}$ & $\begin{array}{c}\mathrm{N}^{\circ} \text { trabajos } \\
\text { JCR }\end{array}$ & Ratio \\
\hline Economía Aplicada & 14 & 124 & 94 & $75,8 \%$ \\
\hline Comercialización e Investigación de Mercados & 13 & 98 & 80 & $81,6 \%$ \\
\hline Organización de Empresas & 9 & 64 & 47 & $73,4 \%$ \\
\hline $\begin{array}{l}\text { Métodos Cuantitativos para Economía y } \\
\text { Empresa }\end{array}$ & 2 & 19 & 12 & $63,2 \%$ \\
\hline Psicología Social & 3 & 9 & 7 & $77,8 \%$ \\
\hline
\end{tabular}

Fuente: elaboración propia a partir de Scopus (2014).

Por otra parte, se pueden ver los datos de publicaciones de autores prolíficos españoles por las instituciones a las que se adscriben. Así, la tabla 6 recoge estos datos ordenados por el número de publicaciones en JCR de cada universidad. Se han seleccionado las diez 
primeras instituciones con mayor número de trabajos publicados. Solo las ocho primeras universidades poseen al menos dos autores prolíficos. Se observa que la Universidad de Islas Baleares destaca ampliamente tanto en el número de autores prolíficos (9) como en el número de artículos publicados en Scopus (83) y en JCR (65). Le sigue la Universidad de Alicante, con cinco autores que han publicado 56 artículos, de los cuales 43 están indexados en JCR. A una gran distancia de estas dos universidades se encuentran el resto de instituciones. En los datos de la tabla cabe destacar que la Universidad de Sevilla ostenta el ratio más elevado de publicaciones en JCR respecto al total de publicaciones en Scopus, alcanzando un 94,1\%. No obstante, la Universidad del País Vasco y la Universidad Politécnica de Cataluña están representados por un autor cada una de ellas que han publicado todos sus artículos en JCR.

\section{Tabla 6 \\ TOP 10 DE INSTITUCIONES A LAS QUE SE ADSCRIBEN LOS AUTORES PROLÍFICOS ESPAÑOLES CON PUBLICACIONES JCR}

\begin{tabular}{|l|c|c|c|c|}
\hline \multicolumn{1}{|c|}{ UNIVERSIDADES } & $\begin{array}{c}\mathrm{N}^{\circ} \\
\text { Autores }\end{array}$ & $\begin{array}{c}\mathrm{N}^{\circ} \text { trabajos } \\
\text { Scopus }\end{array}$ & $\begin{array}{c}\mathrm{N}^{\circ} \text { trabajos } \\
\text { JCR }\end{array}$ & Ratio \\
\hline Universidad de Islas Baleares & 9 & 83 & 65 & $78,3 \%$ \\
\hline Universidad de Alicante & 5 & 56 & 43 & $76,8 \%$ \\
\hline Universidad de Granada & 4 & 21 & 17 & $81 \%$ \\
\hline Universidad Autónoma de Madrid & 2 & 19 & 16 & $84,2 \%$ \\
\hline Universidad de Sevilla & 3 & 17 & 16 & $94,1 \%$ \\
\hline Universidad de Valencia & 5 & 19 & 14 & $73,7 \%$ \\
\hline Universidad de Las Palmas de Gran Canaria & 3 & 15 & 13 & $86,7 \%$ \\
\hline Universidad de Cantabria & 2 & 13 & 11 & $84,6 \%$ \\
\hline Universidad del País Vasco & 1 & 9 & 9 & $100 \%$ \\
\hline Universidad Politécnica de Cataluña & 1 & 9 & 9 & $100 \%$ \\
\hline
\end{tabular}

Fuente: elaboración propia a partir de Scopus (2014) y JCR (2014).

El gráfico 1 refleja las posiciones que ocupan las revistas científicas incluidas en JCR en las que los autores prolíficos españoles han publicado desde 2002 hasta 2013. Todas las revistas son originarias del Reino Unidos y Estados Unidos, salvo la revista española Cuadernos de Economía y Dirección de Empresas. Se observa que la revista con mayor número de publicaciones es Tourism Management, con 39 artículos, seguida de Tourism Economics, con 30 artículos, y de Annals of Tourism Research, con 16 artículos. En este listado aparecen nueve revistas especializadas en turismo entre un total de 16 revistas. Las cinco primeras revistas con mayor número de artículos están dedicadas a publicaciones en el ámbito turístico. En torno al 30\% de las publicaciones de los autores españoles de estas cinco revistas principales son de los autores prolíficos. Así ocurre con las revistas Tourism Management, Annals of Tourism Research e International Journal of Hospitality Management. Sin embargo, Journal of Travel Research alcanza más del 37\% de las publicaciones de autores prolíficos, mientras que Tourism Economics se queda en torno al $26 \%$. 


\section{Gráfico 1 \\ REVISTAS JCR DONDE PUBLICAN CON MAYOR FRECUENCIA LOS AUTORES PROLÍFICOS ESPAÑOLES EN TURISMO}

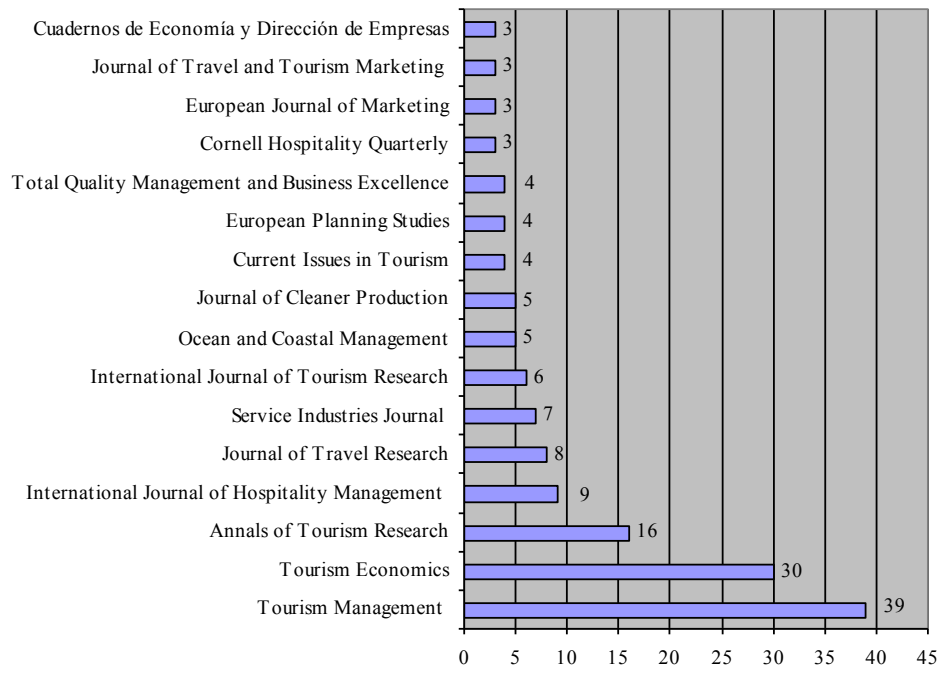

Fuente: elaboración propia a partir de Scopus (2014) y JCR (2014).

En la tabla 7 se han seleccionado los autores prolíficos con siete o más publicaciones incluidas en JCR. De este modo, se puede analizar mediante los cuartiles el nivel de calidad de estas publicaciones en función de las posiciones que ocupan las revistas en sus áreas respectivas. Para ello, se toma como referencia el año de publicación del artículo y el cuartil que ocupó la revista donde fue publicado ese año. A su vez, se realiza una distinción entre las revistas especializadas en turismo y las demás revistas en general. El orden de clasificación se ha establecido en función del número de trabajos publicados en revistas JCR y, en el caso de empate, se ha tenido en cuenta las posiciones que ocupan estas revistas en los cuartiles de JCR.

El primer autor del ranking es J.L. Nicolau, de la Universidad de Alicante y del área de Marketing, que tiene 26 artículos en revistas JCR en el período de estudio, siendo 19 de ellos publicados en revistas especializadas en turismo, lo que representa un $73 \%$ de todos sus trabajos. Además, este autor ha publicado el 34,6\% de sus artículos en revistas situadas en el primer cuartil (Q1). El segundo autor es J. Rosselló, de la Universidad de Islas Baleares y del área de Economía Aplicada, con 20 trabajos en JCR, de los cuales el $70 \%$ se han publicado en revistas especializadas de turismo y el $45 \%$ se han publicado en revistas del primer cuartil. La tercera posición está ocupada por D.M. Frías Jamilena, de la Universidad de Granada y del área de Marketing. Es la primera autora del ranking. Está situada en tercer lugar, con 17 trabajos en JCR y casi todos publicados en revistas especializadas en turismo, estando clasificadas el 29,4\% en el primer cuartil. Otros autores que destacan también en las publicaciones especializadas en revistas de turismo son A. Riera-Font, con el 85,7\% de sus artículos, y J.Alegre, con el 84,6\%. Asimismo, los autores 
más destacados por sus publicaciones en revistas clasificadas en el primer cuartil son J.I. Castillo-Manzano, con el 75\% de sus artículos, seguido de F. Orfila-Sintes $(57,1 \%)$, A.I. Polo-Peña (50\%), H. San Martín (50\%), J. Alegre (46,2\%) y J. Roselló (45\%), Una mención aparte merece E. Claver-Cortés, que es uno de los pioneros en las publicaciones españolas sobre turismo en revistas internacionales. Este autor, junto con sus colaboradores, ha publicado varios artículos en revistas en algunos años previos a ser indexadas en JCR.

Tabla 7

\section{AUTORES PROLÍFICOS ESPAÑOLES SEGÚN SUS PUBLICACIONES POR CUARTILES EN JCR}

\begin{tabular}{|c|c|c|c|c|c|c|c|c|}
\hline \multirow{2}{*}{ Ord. } & \multirow{2}{*}{ Autores } & \multicolumn{2}{|c|}{ Afiliación de autores } & \multirow{2}{*}{$\begin{array}{c}\mathrm{N}^{\circ} \text { artículos } \\
\text { JCR }\end{array}$} & \multicolumn{4}{|c|}{ JCR } \\
\hline & & Centro & Área de conocimiento & & Q1 & Q2 & Q3 & Q4 \\
\hline 1 & J.L. Nicolau & $\begin{array}{l}\text { Universidad de } \\
\text { Alicante }\end{array}$ & $\begin{array}{l}\text { Comercialización e } \\
\text { Investigación Mdos. }\end{array}$ & $\begin{array}{c}26 \\
(19)\end{array}$ & $\begin{array}{l}10 \\
(8)\end{array}$ & $\begin{array}{c}3 \\
(1)\end{array}$ & $\begin{array}{l}10 \\
(8)\end{array}$ & $\begin{array}{c}4 \\
(2) \\
\end{array}$ \\
\hline 2 & J. Rosselló & $\begin{array}{c}\text { Universidad Islas } \\
\text { Baleares } \\
\end{array}$ & Economía Aplicada & $\begin{array}{c}20 \\
(14)\end{array}$ & $\begin{array}{c}9 \\
(3) \\
\end{array}$ & $\begin{array}{c}5 \\
(4) \\
\end{array}$ & $\begin{array}{c}6 \\
(6) \\
\end{array}$ & $\begin{array}{c}0 \\
(0) \\
\end{array}$ \\
\hline 3 & D.M. Frías Jamilena & $\begin{array}{c}\text { Universidad de } \\
\text { Granada }\end{array}$ & $\begin{array}{l}\text { Comercialización e } \\
\text { Investigación Mdos. }\end{array}$ & $\begin{array}{c}17 \\
(16)\end{array}$ & $\begin{array}{c}5 \\
(5) \\
\end{array}$ & $\begin{array}{c}7 \\
(6) \\
\end{array}$ & $\begin{array}{c}5 \\
(5) \\
\end{array}$ & $\begin{array}{c}0 \\
(0) \\
\end{array}$ \\
\hline 4 & E. Claver-Cortés & $\begin{array}{c}\text { Universidad de } \\
\text { Alicante }\end{array}$ & $\begin{array}{c}\text { Organización de } \\
\text { Empresas }\end{array}$ & $\begin{array}{l}16 \\
(7) \\
\end{array}$ & $\begin{array}{c}4 \\
(2) \\
\end{array}$ & $\begin{array}{c}7 \\
(2) \\
\end{array}$ & $\begin{array}{c}3 \\
(3) \\
\end{array}$ & $\begin{array}{c}2 \\
(0) \\
\end{array}$ \\
\hline 5 & M.M. Alonso-Almeida & $\begin{array}{c}\text { Universidad } \\
\text { Autónoma de Madrid }\end{array}$ & $\begin{array}{c}\text { Organización de } \\
\text { Empresas } \\
\end{array}$ & $\begin{array}{l}16 \\
(3) \\
\end{array}$ & $\begin{array}{c}6 \\
(2) \\
\end{array}$ & $\begin{array}{c}2 \\
(0) \\
\end{array}$ & $\begin{array}{c}4 \\
(0) \\
\end{array}$ & $\begin{array}{c}4 \\
(1) \\
\end{array}$ \\
\hline 6 & J.F. Molina-Azorín & $\begin{array}{c}\text { Universidad de } \\
\text { Alicante }\end{array}$ & $\begin{array}{c}\text { Organización de } \\
\text { Empresas }\end{array}$ & $\begin{array}{l}15 \\
(7) \\
\end{array}$ & $\begin{array}{c}4 \\
(2) \\
\end{array}$ & $\begin{array}{c}6 \\
(2) \\
\end{array}$ & $\begin{array}{c}3 \\
(3) \\
\end{array}$ & $\begin{array}{c}2 \\
(0) \\
\end{array}$ \\
\hline 7 & J. Alegre & $\begin{array}{c}\text { Universidad Islas } \\
\text { Baleares } \\
\end{array}$ & Economía Aplicada & $\begin{array}{c}13 \\
(12) \\
\end{array}$ & $\begin{array}{c}6 \\
(6) \\
\end{array}$ & $\begin{array}{c}0 \\
(0)\end{array}$ & $\begin{array}{c}6 \\
(5)\end{array}$ & $\begin{array}{c}1 \\
(1)\end{array}$ \\
\hline 8 & M.A. Rodríguez-Molina & $\begin{array}{c}\text { Universidad de } \\
\text { Granada }\end{array}$ & $\begin{array}{l}\text { Comercialización e } \\
\text { Investigación Mdos. }\end{array}$ & $\begin{array}{l}12 \\
(8) \\
\end{array}$ & $\begin{array}{c}5 \\
(5) \\
\end{array}$ & $\begin{array}{c}4 \\
(0) \\
\end{array}$ & $\begin{array}{c}3 \\
(3) \\
\end{array}$ & $\begin{array}{c}0 \\
(0) \\
\end{array}$ \\
\hline 9 & J. Pereira-Moliner & $\begin{array}{c}\text { Universidad de } \\
\text { Alicante } \\
\end{array}$ & $\begin{array}{c}\text { Organización de } \\
\text { Empresas }\end{array}$ & $\begin{array}{l}12 \\
(5) \\
\end{array}$ & $\begin{array}{c}3 \\
(2) \\
\end{array}$ & $\begin{array}{c}5 \\
(2) \\
\end{array}$ & $\begin{array}{c}2 \\
(1) \\
\end{array}$ & $\begin{array}{c}2 \\
(0) \\
\end{array}$ \\
\hline 10 & J. Rey-Maquieira & $\begin{array}{c}\text { Universidad Islas } \\
\text { Baleares }\end{array}$ & Economía Aplicada & $\begin{array}{l}11 \\
(5) \\
\end{array}$ & $\begin{array}{c}3 \\
(1) \\
\end{array}$ & $\begin{array}{c}2 \\
(0) \\
\end{array}$ & $\begin{array}{c}4 \\
(4) \\
\end{array}$ & $\begin{array}{c}2 \\
(0) \\
\end{array}$ \\
\hline 11 & B. Plaza & $\begin{array}{c}\text { Universidad del País } \\
\text { Vasco } \\
\end{array}$ & Economía Aplicada & $\begin{array}{c}9 \\
(2)\end{array}$ & $\begin{array}{c}4 \\
(1) \\
\end{array}$ & $\begin{array}{c}2 \\
(0)\end{array}$ & $\begin{array}{c}1 \\
(0)\end{array}$ & $\begin{array}{c}2 \\
(1) \\
\end{array}$ \\
\hline 12 & J.A. Jiménez & $\begin{array}{c}\text { Univ. Politécnica de } \\
\text { Cataluña }\end{array}$ & Ingeniería Hidráulica & $\begin{array}{c}9 \\
(0)\end{array}$ & $\begin{array}{c}2 \\
(0)\end{array}$ & $\begin{array}{c}3 \\
(0)\end{array}$ & $\begin{array}{c}3 \\
(0) \\
\end{array}$ & $\begin{array}{c}1 \\
(0) \\
\end{array}$ \\
\hline 13 & J.I. Castillo-Manzano & $\begin{array}{c}\text { Universidad de } \\
\text { Sevilla }\end{array}$ & Economía Aplicada & $\begin{array}{c}8 \\
(4) \\
\end{array}$ & $\begin{array}{c}6 \\
(4)\end{array}$ & $\begin{array}{c}1 \\
(0)\end{array}$ & $\begin{array}{c}0 \\
(0)\end{array}$ & $\begin{array}{c}1 \\
(0) \\
\end{array}$ \\
\hline 14 & A.I. Polo-Peña & $\begin{array}{c}\text { Universidad de } \\
\text { Granada }\end{array}$ & $\begin{array}{l}\text { Comercialización e } \\
\text { Investigación Mdos. }\end{array}$ & $\begin{array}{c}8 \\
(6) \\
\end{array}$ & $\begin{array}{c}4 \\
(4) \\
\end{array}$ & $\begin{array}{c}2 \\
(0) \\
\end{array}$ & $\begin{array}{c}2 \\
(2) \\
\end{array}$ & $\begin{array}{c}0 \\
(0) \\
\end{array}$ \\
\hline 15 & H. San Martín & $\begin{array}{c}\text { Universidad de } \\
\text { Cantabria } \\
\end{array}$ & $\begin{array}{l}\text { Comercialización e } \\
\text { Investigación Mdos. }\end{array}$ & $\begin{array}{c}8 \\
(6) \\
\end{array}$ & $\begin{array}{c}4 \\
(3) \\
\end{array}$ & $\begin{array}{c}1 \\
(1) \\
\end{array}$ & $\begin{array}{c}2 \\
(2) \\
\end{array}$ & $\begin{array}{c}1 \\
(0) \\
\end{array}$ \\
\hline 16 & I. Rodríguez del Bosque & $\begin{array}{c}\text { Universidad de } \\
\text { Cantabria } \\
\end{array}$ & $\begin{array}{l}\text { Comercialización e } \\
\text { Investigación Mdos. }\end{array}$ & $\begin{array}{c}8 \\
(6) \\
\end{array}$ & $\begin{array}{c}3 \\
(2) \\
\end{array}$ & $\begin{array}{c}1 \\
(1) \\
\end{array}$ & $\begin{array}{c}3 \\
(3) \\
\end{array}$ & $\begin{array}{c}1 \\
(0) \\
\end{array}$ \\
\hline 17 & J.A. Castañeda-García & $\begin{array}{l}\text { Universidad de } \\
\text { Granada }\end{array}$ & $\begin{array}{l}\text { Comercialización e } \\
\text { Investigación Mdos. }\end{array}$ & $\begin{array}{c}8 \\
(3)\end{array}$ & $\begin{array}{c}1 \\
(1)\end{array}$ & $\begin{array}{c}4 \\
(0)\end{array}$ & $\begin{array}{c}3 \\
(2)\end{array}$ & $\begin{array}{c}0 \\
(0)\end{array}$ \\
\hline 18 & J.M. López-Bonilla & $\begin{array}{c}\text { Universidad de } \\
\text { Sevilla }\end{array}$ & $\begin{array}{l}\text { Comercialización e } \\
\text { Investigación Mdos. }\end{array}$ & $\begin{array}{c}8 \\
(5) \\
\end{array}$ & $\begin{array}{c}0 \\
(0)\end{array}$ & $\begin{array}{c}2 \\
(2)\end{array}$ & $\begin{array}{c}5 \\
(2) \\
\end{array}$ & $\begin{array}{c}1 \\
(1) \\
\end{array}$ \\
\hline 18 & L.M. López-Bonilla & $\begin{array}{l}\text { Universidad de } \\
\text { Sevilla }\end{array}$ & $\begin{array}{l}\text { Comercialización e } \\
\text { Investigación Mdos. }\end{array}$ & $\begin{array}{c}8 \\
(5)\end{array}$ & $\begin{array}{c}0 \\
(0)\end{array}$ & $\begin{array}{c}2 \\
(2)\end{array}$ & $\begin{array}{c}5 \\
(2)\end{array}$ & $\begin{array}{c}1 \\
(1)\end{array}$ \\
\hline
\end{tabular}




\begin{tabular}{|c|c|c|c|c|c|c|c|c|}
\hline 20 & F. Orfila-Sintes & $\begin{array}{c}\text { Universidad Islas } \\
\text { Baleares }\end{array}$ & $\begin{array}{l}\text { Organización de } \\
\text { Empresas }\end{array}$ & $\begin{array}{c}7 \\
(2)\end{array}$ & $\begin{array}{c}4 \\
(1)\end{array}$ & $\begin{array}{c}0 \\
(0)\end{array}$ & $\begin{array}{c}1 \\
(1)\end{array}$ & $\begin{array}{c}2 \\
(0)\end{array}$ \\
\hline 21 & R. Rigall-I-Torrent & $\begin{array}{c}\text { Universidad de } \\
\text { Girona }\end{array}$ & \begin{tabular}{|c|} 
Fundamentos Análisis \\
Económico
\end{tabular} & $\begin{array}{r}7 \\
(6) \\
\end{array}$ & $\begin{array}{c}3 \\
(3) \\
\end{array}$ & $\begin{array}{c}1 \\
(0)\end{array}$ & $\begin{array}{c}3 \\
(3) \\
\end{array}$ & $\begin{array}{c}0 \\
(0) \\
\end{array}$ \\
\hline 22 & E. Bigné-Alcañiz & $\begin{array}{l}\text { Universidad de } \\
\text { Valencia }\end{array}$ & $\begin{array}{l}\text { Comercialización e } \\
\text { Investigación Mdos. }\end{array}$ & $\begin{array}{c}7 \\
(7) \\
\end{array}$ & $\begin{array}{c}2 \\
(2) \\
\end{array}$ & $\begin{array}{c}2 \\
(2) \\
\end{array}$ & $\begin{array}{c}2 \\
(2) \\
\end{array}$ & $\begin{array}{c}1 \\
(1) \\
\end{array}$ \\
\hline 23 & E. Ruiz-Ballesteros & $\begin{array}{c}\text { Universidad Pablo de } \\
\text { Olavide }\end{array}$ & Antropología & $\begin{array}{c}7 \\
(2) \\
\end{array}$ & $\begin{array}{c}2 \\
(2)\end{array}$ & $\begin{array}{c}2 \\
(0)\end{array}$ & $\begin{array}{c}2 \\
(0)\end{array}$ & $\begin{array}{c}1 \\
(0) \\
\end{array}$ \\
\hline 24 & M. Santana-Gallego & $\begin{array}{c}\text { Universidad Islas } \\
\text { Baleares }\end{array}$ & Economía Aplicada & $\begin{array}{c}7 \\
(4) \\
\end{array}$ & $\begin{array}{c}2 \\
(2)\end{array}$ & $\begin{array}{c}1 \\
(0)\end{array}$ & $\begin{array}{c}2 \\
(1)\end{array}$ & $\begin{array}{c}2 \\
(1) \\
\end{array}$ \\
\hline 25 & A. Riera-Font & $\begin{array}{c}\text { Universidad Islas } \\
\text { Baleares } \\
\end{array}$ & Economía Aplicada & $\begin{array}{r}7 \\
(6) \\
\end{array}$ & $\begin{array}{c}1 \\
(0)\end{array}$ & $\begin{array}{c}2 \\
(2) \\
\end{array}$ & $\begin{array}{c}4 \\
(4) \\
\end{array}$ & $\begin{array}{c}0 \\
(0) \\
\end{array}$ \\
\hline 26 & V. Ramos & $\begin{array}{c}\text { Universidad Islas } \\
\text { Baleares } \\
\end{array}$ & Economía Aplicada & $\begin{array}{r}7 \\
(4) \\
\end{array}$ & $\begin{array}{c}1 \\
(1) \\
\end{array}$ & $\begin{array}{c}1 \\
(0)\end{array}$ & $\begin{array}{c}4 \\
(4) \\
\end{array}$ & $\begin{array}{c}1 \\
(0) \\
\end{array}$ \\
\hline 27 & M.J. Such Devesa & $\begin{array}{c}\text { Universidad de Alcalá } \\
\text { de Henares }\end{array}$ & Economía Aplicada & $\begin{array}{r}7 \\
(5) \\
\end{array}$ & $\begin{array}{c}1 \\
(1) \\
\end{array}$ & $\begin{array}{c}1 \\
(1) \\
\end{array}$ & $\begin{array}{c}4 \\
(3) \\
\end{array}$ & $\begin{array}{c}0 \\
(0) \\
\end{array}$ \\
\hline 28 & J.I. Pulido-Fernández & Universidad de Jaén & Economía Aplicada & $\begin{array}{r}7 \\
(3) \\
\end{array}$ & $\begin{array}{c}1 \\
(1) \\
\end{array}$ & $\begin{array}{c}1 \\
(0)\end{array}$ & $\begin{array}{c}1 \\
(1) \\
\end{array}$ & $\begin{array}{c}4 \\
(1) \\
\end{array}$ \\
\hline 29 & J.M. Peiró & $\begin{array}{c}\text { Universidad de } \\
\text { Valencia }\end{array}$ & Psicología Social & $\begin{array}{c}7 \\
(0) \\
\end{array}$ & $\begin{array}{c}1 \\
(0)\end{array}$ & $\begin{array}{c}1 \\
(0)\end{array}$ & $\begin{array}{c}1 \\
(0)\end{array}$ & $\begin{array}{c}4 \\
(0) \\
\end{array}$ \\
\hline 30 & D.R. Medina-Muñoz & $\begin{array}{l}\text { Univ. de Las Palmas } \\
\text { de Gran Canaria }\end{array}$ & $\begin{array}{c}\text { Organización de } \\
\text { Empresas }\end{array}$ & $\begin{array}{c}7 \\
(5) \\
\end{array}$ & $\begin{array}{c}1 \\
(0)\end{array}$ & $\begin{array}{c}0 \\
(0) \\
\end{array}$ & $\begin{array}{c}3 \\
(3) \\
\end{array}$ & $\begin{array}{c}3 \\
(2) \\
\end{array}$ \\
\hline 30 & R.D. Medina-Muñoz & $\begin{array}{l}\text { Univ. de Las Palmas } \\
\text { de Gran Canaria }\end{array}$ & $\begin{array}{c}\text { Organización de } \\
\text { Empresas }\end{array}$ & $\begin{array}{r}7 \\
(5) \\
\end{array}$ & $\begin{array}{c}1 \\
(0) \\
\end{array}$ & $\begin{array}{c}0 \\
(0) \\
\end{array}$ & $\begin{array}{c}3 \\
(3) \\
\end{array}$ & $\begin{array}{c}3 \\
(2) \\
\end{array}$ \\
\hline 32 & V. Martínez-Tur & $\begin{array}{l}\text { Universidad de } \\
\text { Valencia }\end{array}$ & Psicología Social & $\begin{array}{c}7 \\
(0) \\
\end{array}$ & $\begin{array}{c}0 \\
(0) \\
\end{array}$ & $\begin{array}{c}2 \\
(0) \\
\end{array}$ & $\begin{array}{c}1 \\
(0)\end{array}$ & $\begin{array}{c}4 \\
(0) \\
\end{array}$ \\
\hline
\end{tabular}

Fuente: elaboración propia a partir de Scopus (2014) y JCR (2014).

En este ranking se concentra el $84,4 \%$ de autores en las tres áreas de conocimiento principales, como son: Comercialización e investigación de Mercados (10 autores), Economía Aplicada (10 autores) y organización de Empresas (7 autores). Asimismo, el 78,1\% de los autores pertenecen a siete instituciones, destacando la Universidad de Islas Baleares, con siete autores; siguiéndole las universidades de Alicante y Granada, con cuatro autores cada una de ellas; las universidades de Sevilla y Valencia, con tres autores; y las universidades de Las Palmas de Gran Canaria y Cantabria, con dos autores cada una de ellas.

Se puede ver que hay un número ligeramente superior de autores prolíficos que publican en revistas especializadas en turismo (18 autores) de los que publican en otras revistas (13 autores). Un autor publicó un número igual de artículos en ambos tipos de revistas. En este sentido, se pueden mencionar algunos de los autores que concentran mayormente sus publicaciones en revistas del ámbito turístico, como son los siguientes: E. Bigné, con todos sus 7 artículos publicados en revistas sobre turismo; D.M. Frías-Jamilena, con 16 de sus 17 artículos; y J. Alegre, con 12 de sus 13 artículos. Por el contrario, hay tres autores que no han publicado ningún trabajo en revistas especializados en turismo, como son J.A. Jiménez, V. Martínez-Tur y J.M. Peiró.

Por otro lado, si se observa la posición de las revistas científicas, atendiendo a la clasificación de cuartiles, los autores prolíficos han publicado principalmente en revistas JCR que están especializadas en turismo y clasificadas en el cuartil Q1, con un total de 324 artículos; en segundo término, en revistas no especializadas en turismo clasificadas en Q1, 
con 184 artículos; y en tercer lugar lo ocupan las publicaciones en revistas sobre turismo y clasificadas en Q2, con 100 artículos. En general, en todo tipo de revistas, los autores con más trabajos publicados en Q1 son J.L. Nicolau, con 10 artículos, y J. Rosselló, con 9 artículos. Pero en revistas sobre turismo destaca en primer lugar J.L. Nicolau, con 8 artículos, seguido de J. Alegre, con 6 artículos, y D.M. Frías, con 5 artículos.

En cuanto al género, hay un total de ocho autoras entre los 32 autores del ranking que, además de la citada anteriormente (D.M. Frías Jamilena), son las siguientes: M.M. Alonso-Almeida, A.I. Polo-Peña, B. Plaza, F. Orfila-Sintes, M.J. Such Devesa, M. Santana-Gallego y R.D. Medina-Muñoz. La autora F. Orfila-Sintes es quien posee un mayor porcentaje de artículos publicados en revistas situadas en el primer cuartil, con un $57,1 \%$ de ellos, siendo la mayor parte de sus trabajos publicados en otras revistas distintas al ámbito turístico. D.M. Frías y A.I. Polo-Peña son las autores que más publicaron en revistas especializadas en turismo, con un $94,1 \%$ y $75 \%$, respectivamente.

El número de autores por trabajo publicado es también una referencia interesante a observar en relación con su productividad. En el gráfico 2 se aprecian estas cifras en función de las colaboraciones que existen entre autores en su producción científica. La media aritmética es de 2,68 autores por artículo publicado, siendo casi igual el número de trabajos publicados con dos autores $(37,44 \%)$ que con tres autores $(36,56 \%)$. Poco más del $10 \%$ de los artículos se publica en solitario y está próximo a este porcentaje las colaboraciones entre cuatro autores. En torno al 5\% de los trabajos han sido publicados por cinco o más autores. Se pueden citar también algunos autores prolíficos destacados por su menor número de colaboraciones necesarias para publicar sus trabajos, como son los siguientes: J.L. Nicolau, que publica con una media de co-autoría de 1,58; B. Plaza, con una media de 1,78 autores por artículo; J.I. Castillo-Manzano, J.M. López-Bonilla y L.M. López-Bonilla, con una media de 2,13 autores cada uno de ellos; E. Ruiz-Ballesteros, con 2,14 autores; H. San Martín, con 2,25 autores; J.I. Pulido-Fernández, con 2,29 autores; I. Rodríguez del Bosque, con 2,37 autores; y J. Rosselló, con 2,38 autores.

\section{Gráfico 2 \\ COLABORACIONES POR ARTÍCULO DE LOS AUTORES PROLÍFICOS ESPAÑOLES EN PUBLICACIONES JCR}

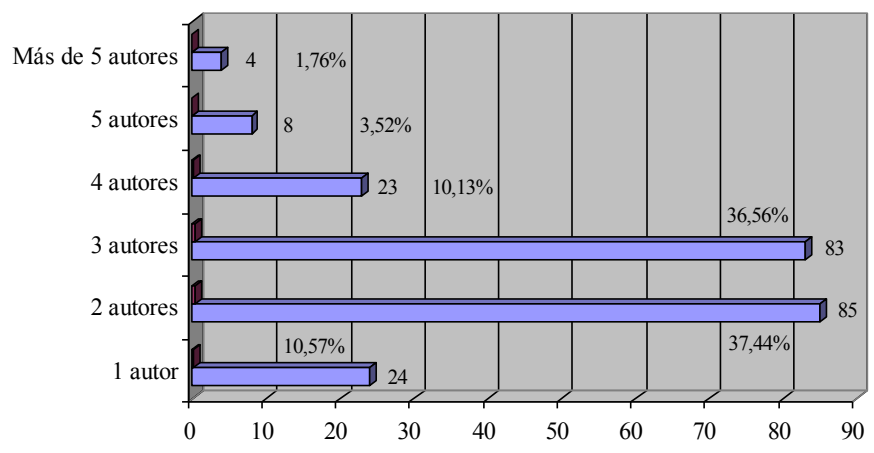

Fuente: elaboración propia a partir de Scopus (2014). 
Las colaboraciones mixtas con autores extranjeros y autores de otras áreas e instituciones son escasas. Sin embargo, se pueden citar algunas excepciones, como J. ReyMaquieira, que publica seis de sus once artículos en JCR en colaboración con autores extranjeros. En concreto, este autor ha publicado con dos autores brasileños, dos autores tailandeses y un autor australiano. Por su parte, M.M. Alonso-Almeida destaca por sus colaboraciones con otras instituciones españolas, habiendo participado con autores de las Universidades de Barcelona, Girona, Pompeu Fabra e Internacional de Cataluña, además de dos universidades pertenecientes a México y Alemania. Por último, J.I. Pulido-Fernández (adscrito al área de Economía Aplicada) resalta sobre todo porque ha publicado con varios autores españoles de distintas áreas de conocimiento a la suya propia, como son Métodos Cuantitativos para la Economía y la Empresa y Comercialización e Investigación de Mercados.

Otro indicador relevante en la productividad de los autores es el número de citas recibidas por sus publicaciones. En este sentido, si se observan las citas indicadas en Scopus de los trabajos publicados por los autores prolíficos españoles analizados, hay que distinguir a cuatro primeros autores que superan el medio millar de citas por sus publicaciones en JCR, que son los siguientes: J.M. Peiró, con 633 citas; E. ClaverCortés, con 626 citas; J.F. Molina-Azorín, con 589 citas; J. Alegre, con 542 citas. Le siguen otros autores destacados, como son J. Rosselló, con 471 citas; E. Bigné, con 437 citas; H. San Martín, con 435 citas; I. Rodríguez del Bosque, con 414 citas; J.L Nicolau, con 384 citas; J. Pereira-Moliner, con 353 citas; y F. Orfila-Sintes, con 332 citas, que precisamente es la primera autora con más número de citas. Lógicamente, el número de citas está muy influido por el número de trabajos y por los años que han transcurrido desde su publicación, además del impacto de la propia revista en la que se publica. En este sentido, J.M. Peiró alcanza un número de citas por trabajo de 90,4 debido a que uno de sus artículos tiene una gran cantidad de citas. A continuación, cabe mencionar a otros autores cuyas publicaciones registran un número de citas en torno a la cincuentena o superior, como ocurre con E. Bigné, con 62,4 de citas medias; H. San Martín $(54,4)$, I. Rodríguez del Bosque $(51,8)$ y F. Orfila-Sintes $(47,4)$.

En la tabla 8 se detallan los trabajos publicados de los autores analizados que han recibido un mayor número de citas en Scopus. Esta tabla recoge los 25 trabajos que han superado poco más de las 50 citas. El primer trabajo sobresale ampliamente, alcanzando las 509 citas, siendo publicado en 2005 por J.M. Peiró como tercer autor conjuntamente con otros dos autores españoles más. El siguiente trabajo más citado es de E. Bigné, que aparece como primer autor del mismo y acompañado de otros dos autores más. A continuación, le siguen dos artículos publicados en el año 2008 por los mismos autores: I. Rodríguez del Bosque y H. San Martín. Otros autores destacados en este sentido son los siguientes: J. Alegre es el autor que posee un mayor número de artículos en este listado, apareciendo cinco veces entre estos 25 artículos más citados; E. Bigné, E. Claver-Cortés, J.F. Molina-Azorín, F. Orfila-Sintes, J. Rosselló y H. San Martín son autores que aparecen en tres artículos de este listado. Además, hay que resaltar que 18 de los 25 artículos citados en esta tabla se han publicado en las tres revistas científicas principales del ámbito turístico, como son: Tourism Management (con 9 artículos), Annals of Tourism Research (con 7 artículos) y Journal of Travel Research (con 2 artículos). 


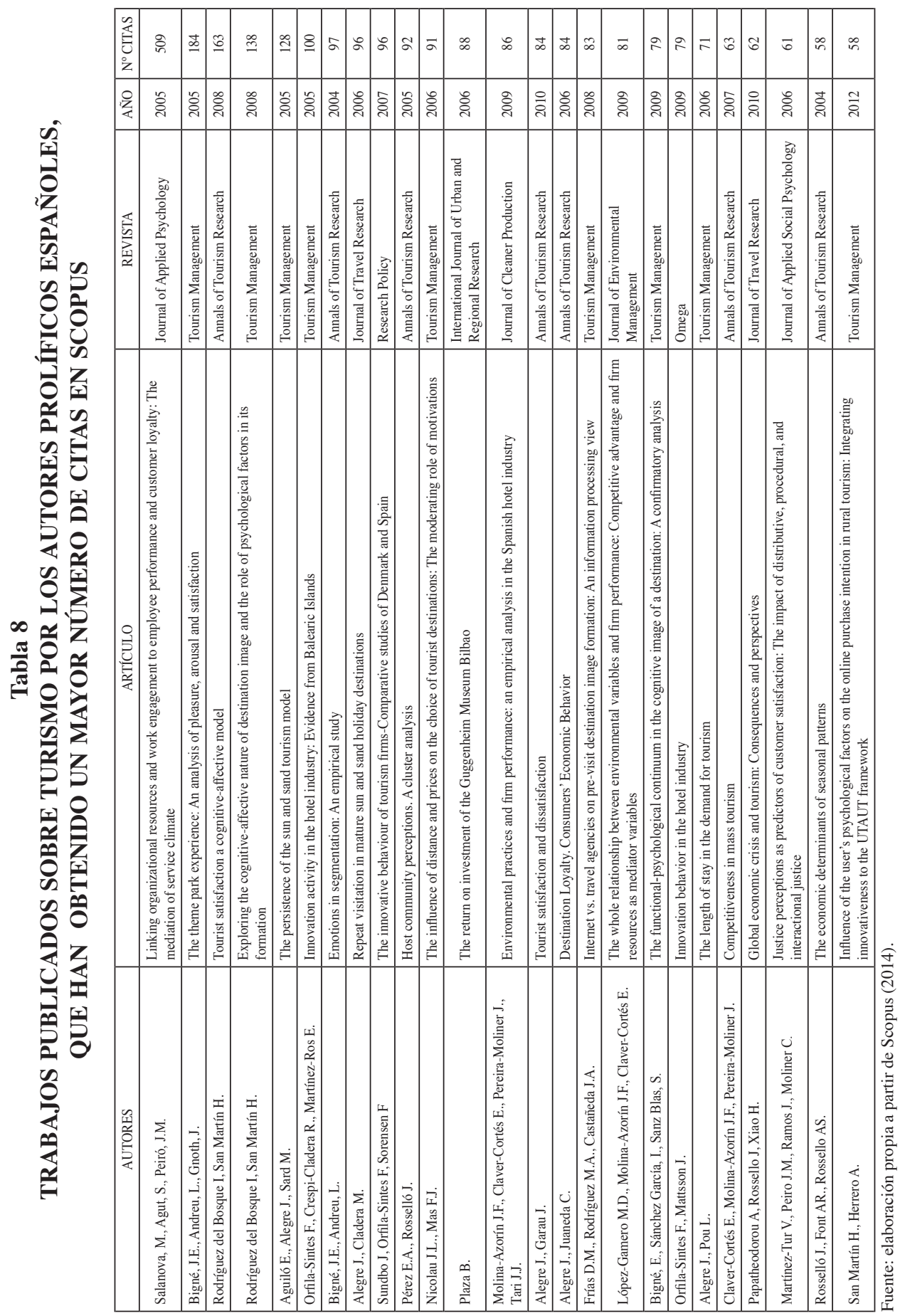




\section{CONCLUSIONES}

En el presente estudio se han analizado las publicaciones sobre turismo en revistas con impacto internacional por parte de investigadores españoles durante el período comprendido entre 2002 y 2013. La nueva legislación sobre universidades ha afectado en gran medida a la producción científica española, en general, así como a la investigación turística en concreto, dado que hay un crecimiento continuo en el número de publicaciones desde el año 2002 hasta la actualidad.

Las revistas en las que publican los autores españoles son muy diversas, incluyendo revistas especializadas en turismo y revistas de otros ámbitos de estudio. Sin embargo, las revistas principales de turismo son las más destacadas en número de publicaciones de autores españoles. En los últimos años se han incorporado cada vez más revistas científicas especializadas en turismo a las bases de datos Scopus y, sobre todo, Web of Science, por lo que es muy probable que haya un mayor aumento de publicaciones en este tipo de revistas en el futuro. Por ello, un análisis bibliométrico sobre la investigación turística debe contemplar el estudio de revistas científicas específicas de cualquier área, así como otras revistas de carácter general. Como indican Corral y Cànoves (2013), el peso de las revistas no turísticas afecta a la representatividad de la mayoría de los estudios bibliométricos.

Llama la atención que las revistas españolas más utilizadas por autores españoles en la investigación turística pertenezcan al área de Geografía, siendo cuatro de las siete revistas más destacadas en Scopus, de las que sobresale el Boletín de la Asociación de Geógrafos Españoles con un mayor número de publicaciones de autores españoles. Sin embargo, con la reciente aparición en Scopus de la única revista española especializada en turismo, denominada Cuadernos de Turismo, es previsible que ocupe una de las primeras posiciones en este ranking de revistas por autores españoles en los próximos años. No cabe duda que la aparición de esta revista científica española en el panorama internacional es una gran noticia para el mundo académico, que es deseable que se afiance y que se extienda su ejemplo a otras revistas españolas, para que un país con tanto liderazgo turístico pueda estar mejor representado no solo por sus investigadores sino también por sus propias revistas científicas especializadas en turismo.

Se ha identificado como autores prolíficos españoles a un total de 46 autores que han publicado, al menos, seis trabajos en revistas indexadas en revistas incluidas en el Journal Citation Reports (JCR) y en el ámbito de la investigación turística. La casi totalidad de estos autores publica un porcentaje mayor de artículos en revistas JCR que en revistas indexadas en Scopus únicamente. Existe una gran concentración de las áreas de conocimiento a las que están adscritos estos autores españoles, como son: Economía Aplicada, Comercialización e Investigación de Mercados y Organización de Empresas. Así pues, las áreas de los autores prolíficos se concentran en gran parte en los ámbitos de la economía y la empresa. También destacan algunas instituciones a las que pertenecen los autores españoles, como pueden ser, especialmente, las universidades de Islas Baleares y de Alicante. Las revistas científicas preferentes de los autores prolíficos españoles son Tourism Management y Tourism Economics. Asimismo, en el ámbito territorial, los resultados indican que la producción científica se concentra especialmente en las regio- 
nes de carácter más turístico, en consonancia con estudios previos (v.g., López-Bonilla y López-Bonilla, 2008; Albacete-Sáez y Fuentes-Fuentes, 2010; Vargas, 2011a; Albacete, Fuentes y Haro-Domínguez, 2013).

El ranking de autores está liderado claramente por J.L. Nicolau (Universidad de Alicante), sobre todo, en lo que respecta a la cantidad de trabajos publicados, con 26 artículos publicados en Scopus, así como por conseguir publicar el mayor número de artículos en revistas JCR clasificadas en el cuartil Q1 entre todos los autores prolíficos, con 10 artículos. Pero también es el autor que más publicaciones ha logrado en revistas especializadas en turismo. Este investigador aparece también entre los primeros del ranking de autores en otros estudios previos (Albacete-Sáez y Fuentes-Fuentes, 2010; Hernández, Campón y Folgado, 2011; Albacete, Fuentes y Haro-Domínguez, 2013). El segundo lugar del ranking lo ocupa J. Rosselló (Universidad Islas Baleares), con 21 trabajos publicados, siendo ocho de ellos publicaciones en JCR. En la tercera posición por número de trabajos publicados en Scopus aparece la primera autora, D.M. Frías Jamilena (Universidad de Granada). Cabe señalar que hay ocho mujeres entre los 32 autores prolíficos principales que han publicado en JCR. Esto supone un $25 \%$ del total de autores de este ranking que, aunque no alcanza una supuesta paridad, no es tampoco muy bajo si se consideran los dos únicos análisis de autoría que reflejan estos datos en estudios previos, tanto en el ámbito nacional como internacional. Así, en el estudio de Zhao y Ritchie (2007) se indica que hay un 10,5\% de mujeres en su ranking internacional de autores, mientras que en el estudio más reciente de Albacete, Fuentes y Haro-Domínguez (2013) se observa que la representación femenina española alcanza al $16,7 \%$.

Los autores prolíficos españoles están todavía a una cierta distancia de los autores internacionales en la investigación turística. En la totalidad de revistas JCR analizadas, sean o no especializadas en turismo, se han detectado diez autores que han publicado diez o más artículos. Sin embargo, los ranking de autores internacionales superan ampliamente estas cifras. Así, por ejemplo, Ryan (2005) establece un ranking de 52 autores prolíficos, basado en el análisis de 16 revistas líderes en turismo durante el período de 1990 a 2004, observando que, entre las tres revistas JCR incluidas en este listado en esas fechas (Annals of Tourism Research, Journal of Travel Research y Tourism Management), se hallan 18 autores que han publicado, al menos, diez artículos. En este sentido, Zhao y Ritchie (2007) identifican 57 autores prolíficos a través de un estudio de ocho revistas sobre turismo entre 1985 y 2004, encontrando que 38 autores publicaron un mínimo de diez trabajos en las tres revistas JCR mencionadas anteriormente. En el estudio de Park, Phillips, Canter y Abbott (2011) se descubre también que hay 28 autores líderes con diez o más publicaciones durante los años 2000 a 2009 en esas tres revistas.

La productividad científica de los autores se puede medir de manera muy variada a través de distintos aspectos y niveles. Uno de ellos es publicar en revistas de alto impacto como son las publicaciones indexadas en JCR. Por ello, se ha identificado en este trabajo un ranking de autores prolíficos españoles en investigación turística que publican en este tipo de revistas. Pero también se puede observar que hay distintos niveles de productividad dentro de estas mismas revistas JCR si se atiende a su clasificación por factor de impacto y por cuartiles. Esto supone que es posible clasificar a los autores según la posición que ocupa la revista en la que ha publicado. Además, la 
productividad se puede medir también a través del número de autores que colaboran en cada trabajo publicado, así como a través del número de citas que recibe cada artículo. En el presente trabajo se ha pretendido dar una visión general de todos estos aspectos en torno a la investigación turística española.

En cuanto a las citas recibidas de las publicaciones realizadas por los autores prolíficos españoles en el ámbito turístico y en revistas indexadas en el JCR, el tiempo es lógicamente un factor clave para aumentar estas cifras. Los artículos más citados tienen una antigüedad que supera los seis años. Asimismo, la mayoría de los artículos más citados de los autores prolíficos españoles se han publicado en las revistas científicas más prestigiosas en el ámbito turístico en la actualidad, como son Tourism Management y Annals of Tourism Research y, en menor medida, Journal of Travel Research. Estas tres revistas conforman la terna de publicaciones sobre turismo de mayor trayectoria en el Journal Citation Reports. A este respecto, los trabajos publicados en revistas especializadas en turismo alcanzan una mejor posición por cuartiles que las otras revistas. Esto quiere decir que los autores prolíficos pueden obtener un mayor rendimiento bibliométrico de sus publicaciones en revistas sobre turismo. Es decir, estos autores son capaces de publicar trabajos sobre turismo en revistas con mayor impacto cuando pertenecen al ámbito turístico. Actualmente, otras revistas sobre investigación turística se han incorporado a esta base de datos internacional, por lo que habrá que seguirlas en el futuro para conocer su rendimiento.

Por otro lado, las tres cuartas partes de los trabajos se han publicado entre dos o tres autores, siendo la media de 2,68 autores por artículo, siendo muy similar a la media de 2,73 que se recoge en el estudio de Corral-Marfil, Rodríguez, Vargas y Cànoves (2015), centrándose en Cataluña. Estas cifras resultan algo elevadas en comparación con la media de 2,1 autores que indican Zhao y Ritchie (2007) en un contexto internacional. En este sentido, vuelve a destacar el nombre de J.L. Nicolau, con una media de 1,58 autores por artículo en el conjunto de sus publicaciones. De acuerdo con Ardenuy (2012), la colaboración científica puede ser local (entre investigadores de la misma institución), doméstica (entre investigadores nacionales de distintas instituciones) e internacional (entre investigadores de distintos países). En el presente estudio, la colaboración entre autores e instituciones es predominantemente local y unidisciplinaria. Es decir, la gran mayoría de autores prolíficos españoles publican sus trabajos con autores que pertenecen a la misma institución y a la misma área de conocimiento científico. Dado que éste es un estudio que trata de recoger una primera etapa de consolidación en la difusión internacional de los autores españoles, es muy posible que se tienda a colaborar más abiertamente con otros autores, áreas de conocimiento, instituciones y países distintos en los próximos años. Sería interesante que se pudieran establecer más contactos entre estos autores prolíficos de distintas instituciones para desarrollar algunos trabajos y proyectos en común.

Una última consideración merece nuevamente el hecho de darse una concentración tan elevada de los autores prolíficos españoles en unas pocas áreas de conocimiento a las que están adscritos. Esto debe ser motivo para una reflexión más profunda que hay que extenderla al ámbito de la política universitaria en general. Tan solo unas pocas áreas de conocimiento del conjunto de las ciencias sociales y jurídicas, así como de las humanidades, aparecen en estos ranking de autores a nivel internacional. La categoría de turismo de las publicaciones de JCR ha ampliado su número de revistas en los últimos años, pero 
todavía es relativamente escaso, y se trata de un campo de estudio multidisciplinar. No obstante, el presente trabajo recoge todas las publicaciones de autores españoles, tanto en revistas especializadas en turismo como aquellas que están especializadas en cualquier otro ámbito de estudio o tienen un carácter general.

Todo ello puede llevar a pensar que existe una mayor presión por publicar en revistas relevantes en algunas áreas de conocimiento científico. Es relativamente fácil comprobarlo a través de los criterios que se exigen por la Agencia Nacional de Evaluación de la Calidad y Acreditación (ANECA) para la promoción de los cuerpos docentes universitarios, así como por la Comisión Nacional Evaluadora de la Actividad Investigadora (CNEAI) para la obtención de los sexenios de investigación. Se puede observar que hay disciplinas, áreas de conocimiento o campos de estudio con criterios mucho más laxos que otros. De hecho, unos investigadores pueden obtener sus sexenios sin tener ningún artículo indexado en JCR y otros tienen que alcanzarlos con todos sus artículos indexados en JCR o, al menos, que alguno o algunos de ellos estén publicados en revistas consideradas de máximo nivel, como estar clasificada en el cuartil Q1, y que además pertenezcan a la especialidad científica de su autor. En el mismo sentido, es muy posible también que un profesor titular consiga acreditarse a catedrático de universidad sin apenas haber publicado algún artículo en revistas JCR de menor impacto y, en cambio, otros menos afortunados no pueden ser acreditados con una veintena de artículos en revistas JCR de impactos variados. Todo ello, considerando que el currículum investigador, aparte de otras actividades, se mide principalmente por las publicaciones realizadas y, sobre todo, que hayan sido en revistas científicas indexadas. En definitiva, los niveles de exigencia investigadora son muy desiguales entre las distintas áreas de conocimiento que se ocupan del turismo, especialmente en las ciencias sociales y jurídicas y humanidades, por lo que sería bastante sensato encontrar un punto de equilibrio en el que se favorezcan los esfuerzos personales y globales de la comunidad científica española que trabaja sobre el mismo objeto de estudio.

Para futuros estudios se podría profundizar en el análisis de la productividad de la investigación turística española y, para ello, sería muy útil emplear otros indicadores bibliométricos, como el número de publicaciones fraccionadas por autor y el índice $\mathrm{h}$, que está basado en el número de trabajos publicados por un autor que ha alcanzado un número h de citas cada una de ellos. Asimismo, habría que seguir analizando el conjunto de publicaciones relativas a la investigación turística para descubrir sus pautas de evolución en los próximos años. También sería de gran interés investigar las colaboraciones en redes que conforman los autores más productivos.

En definitiva, ha habido relativamente pocos estudios bibliométricos centrados en la investigación turística española hasta la fecha. Sobre todo, son muy escasos los análisis de autoría. Dada la posición que ocupa España en el ámbito turístico mundial, este tipo de estudios puede ser de interés para la comunidad científica especializada en turismo, así como para los gestores públicos vinculados con este ámbito de estudio, ofreciendo la oportunidad de profundizar en el conocimiento de la actividad investigadora que desarrollan los autores o investigadores españoles. Además, este trabajo puede ser de utilidad para distintos grupos de interés, como son los propios investigadores y las instituciones académicas, así como los estudiantes de postgrado y doctorado. 


\section{BIBLIOGRAFÍA}

AGENCIA VALENCIANA DE TURISMO (2006): “Análisis de las tesis doctorales de turismo realizadas en universidades españolas", Papers de Turisme, n 40, pp. 111-116.

AGUILÓ, E. (2010): “Una panorámica de la economía del turismo en España”, Cuadernos de Economía, vol. 33, n 91, pp. 5-42.

ALBACETE-SÁEZ, C.A. y FUENTES-FUENTES, M.M. (2010): "Difusión de la investigación española sobre turismo en revistas internacionales", Revista de Análisis Turístico, no 9, pp. 14-29.

ALBACETE, C.A., FUENTES, M.M. y HARO-DOMÍNGUEZ, M.C. (2013): “La investigación española en turismo con impacto internacional (1997-2011). Una perspectiva de la economía y la dirección de la empresa", Cuadernos de Economía y Dirección de la Empresa, $\mathrm{n}^{\circ} 16$, pp. 17-28.

ANTÓN I CLAVÉ, S., LÓPEZ PALOMEQUE, F., MARCHENA GÓMEZ, M.J. y VERA REBOLLO, J.F. (1996): "La investigación turística en España: aportaciones de la Geografía (1960-1995)”, Estudios Turísticos, n 129, pp. 165-208.

ARDANUY, J. (2012): "Scientific collaboration in Library and Information Science viewed through the Web of Knowledge: the Spanish case", Scientometrics, vol. 90, $\mathrm{n}^{\mathrm{o}} 3$, pp. 877-890.

BIGNÉ ALCAÑIZ, J.E., ANDREU SIMÓ, L., SÁNCHEZ GARCÍA, I. y ALVARADO HERRERA, A. (2008): "Investigación internacional en marketing turístico: análisis de contenido sobre temas y metodologías", PASOS. Revista de Turismo y Patrimonio Cultural, vol. 6, n 3, pp. 391-398.

BOTE GÓMEZ, V. (1996): "La investigación en España sobre turismo y desarrollo económico", Estudios Turísticos, n 129, pp. 9-22.

CAMISÓN ZORNOZA, C. y MONFORT MIR, V.M. (1996): "La calidad en el turismo español: balance y prospectiva de la investigación", Estudios Turísticos, n ${ }^{\circ}$ 128, pp. 129-161.

CAMISÓN ZORNOZA, C., BOU LLUSAR, J.C., ROCA, V. y MONTESINOS, C. (1997): "Enlace de la investigación en turismo y en gestión de la calidad: balance de una década", Papers de Turisme, n 20, pp. 19-38.

CEBAllos, C., ARIAS, C., RUIZ, A., SANZ, C. y VÁZQUEZ, I. (2010): "La formación en turismo en España: pasado, presente y futuro en el nuevo Espacio Europeo de Educación Superior", Cuadernos de Turismo, no 25, pp. 45-67.

CODINA-CANET, M.A., OLMEDA-GÓMEZ, C. y PERIANES-RODRÍGUEZ, A. (2013): “Análisis de la producción científica y de la especialización temática de la Universidad Politécnica de Valencia. Scopus (2003-2010)”, Revista Española de Documentación Científica, vol. 36, n $\mathrm{n}^{\mathrm{3}}$, pp. 1-17.

CORRAL-MARFIL, J.A. (2013): "Estudio bibliométrico de las Actas del Congreso de la Asociación Española de Expertos Científicos en Turismo (AECIT, 1994-2012)", Revista de Análisis Turístico, vol. 16, n² 2, pp. 33-44.

CORRAL, J.A. y CÀNOVES, G. (2013): "La investigación turística publicada en revistas turísticas y no turísticas: análisis bibliométrico de la producción de las universidades catalanas", Cuadernos de Turismo, no 31, pp. 55-81. 
CORRAL, J.A. y CÀNOVES, G. (2014): “La colaboración científica en turismo: grupos y redes de investigación en Cataluña", Documents d'Anàlisi Geogràfica, vol. 60, n ${ }^{\circ}$ 1, pp. 57-86.

CORRAL, J.A. y SAN EUGENIO, J. de (2013): "La investigación de la geografía del turismo en España a través del análisis bibliométrico de las actas del Coloquio de Geografía del Turismo, Ocio y Recreación (1990-2012)" Boletín de la Asociación de Geógrafos Españoles, n ${ }^{\circ}$ 63, pp. 535-549.

CORRAL-MARFIL, J.A., RODRÍGUEZ, I.M., VARGAS, A. y CÀNOVES, G. (2015): "Estudio de la investigación turística a través de las coautorías de artículos: cálculo de indicadores de colaboración y análisis de redes sociales. El caso de las universidades catalanas", PASOS. Revista de Turismo y Patrimonio Cultural, vol. 13, n 4, pp. 789-803.

ESTEBAN, A. (2000): "La investigación turística en la universidad española", Estudios Turísticos, ${ }^{\circ}$ 144-145, pp. 155-180.

FIGUEROLA PALOMO, M. (2000): “Los trabajos de investigación de la economía del turismo en el Instituto de Estudios Turísticos: (1974-1993)", Estudios Turísticos, n ${ }^{\circ}$ 144-145, pp. 17-45.

GONZÁlEZ-ALBO, B., MORENO, L., APARICIO, J., MORILLO, F. y BORDONS, M. (2009): La investigación española sobre turismo a través de publicaciones científicas de difusión nacional e internacional (Web of Science e ISOC 1998-2008). IEDCYT, CCHS, CSIC. Documento no publicado.

GRANDA-ORIVE, J.I., ALONSO-ARROYO, A., GARCÍA-RÍOS, F., SOLANO-REINA, S., JIMÉNEZ-RUIZ, C.A. y ALEIXANDRE-BENAVENT, R. (2013): Revista Española de Documentación Científica, vol. 36, $\mathrm{n}^{\circ}$ 2, pp. 1-9.

HALL, C.M. (2005): "Systems of surveillance and control: commentary on «An analysis of institutional contributors to three major academic tourism journals: 1992-2001»", Tourism Management, vol. 26, $\mathrm{n}^{\circ}$ 5, pp. 653-656.

HERNÁNDEZ, J.M., CAMPÓN, A.M. y FOLGADO, J.A. (2011): "La investigación en turismo en España a través de las revistas de dirección de empresas", Revista de Análisis Turístico, $\mathrm{n}^{\mathrm{o}}$ 12, pp. 1-9.

LÓPEZ-BONILLA, J.M. y LÓPEZ-BONILLA, L.M. (2004): "Evolución y perspectivas del enfoque interdisciplinario en el estudio del turismo", Estudios Turísticos, n 160 , pp. 31-44.

LÓPEZ-BONILLA, J.M. y LÓPEZ-BONILLA, L.M. (2008): "Producción científica española en marketing turístico”, Estudios Turísticos, n 177, pp. 35-50.

LÓPEZ-BONILLA, J.M., GRANADOS-PEREA, C. y LÓPEZ-BONILLA, L.M. (2017): "Primera generación de autores con difusión internacional en la investigación turística española", Revista Española de Documentación Científica, vol. 40, n 3, pp. 1-18.

LOSEKOOT, E., VERGINAS, C.S. y WOOD, C. (2001): "Out for the count: Some methodological questions in publications counting literature", International Journal of Hospitality Management, vol. 20, n 3, pp. 233-244.

McKERCHER, B. (2007): “An analysis of prolific authors", Journal of Hospitality and Tourism Education, vol. 19, n 2, pp. 23-30. 
McKERCHER, B. (2008): "A citation analysis of tourism scholars", Tourism Management, vol. 29, nº 6, pp. 1226-1232.

MINISTERIO DE EDUCACIÓN, CULTURA Y DEPORTE (2017): Estadística de estudiantes universitarios. Disponible en http://www.mecd.gob.es/servicios-al-ciudadanomecd/estadisticas/educacion/universitaria/estadisticas/alumnado.html

MIRALBEL, O., LAMSFUS, C., GOMIS, J.M. y GONZÁLEZ, F. (2012): "Estudio de las TIC y el Turismo en España. Análisis de las ponencias del congreso TURITEC entre 1999 y 2010”. En A. Guevara, A. Aguayo, J.L. Caro y A. Cerezo (Eds.), TuriTec 2012, pp. 498-517. Facultad de Turismo, Universidad de Málaga. Málaga.

MORENO, S. y PICAZO, P. (2012): “Difusión de la investigación científica en revistas de turismo realizada por instituciones españolas", Revista de Análisis Turístico, vol. $14, n^{\circ} 2$, pp. 33-52.

ORTEGA MARTÍNEZ, E. y RODRÍGUEZ HERRÁIZ, B. (2004): "La investigación turística a través de tesis doctorales. Un análisis comparativo entre España y Francia”. Estudios Turísticos, no 159, pp. 5-27.

PARK, K., PHILLIPS, W.J., CANTER, D.D. y ABBOTT, J. (2011): “Hospitality and tourism research rankings by authors, university, and country using six major journals: the first decade of the new millennium", Journal of Hospitality \& Tourism Research, vol. $35, n^{\circ} 3$, pp.381-416.

PELÁEZ-VERDET, A. y FERRERA-BLASCO, M. (2017): "The usefulness of social media analysis within scholarly publications: a study of first-tier tourism journals", Tourism \& Management Studies, vol. 13, n 1, pp. 43-50.

RUIZ, A., CHÁVEZ, E. y ARIAS, C. (2006): "Estado de la investigación de las publicaciones sobre yield management en las revistas de turismo y hostelería", Papers de Turisme, $\mathrm{n}^{\circ} 40$, pp. 7-29.

RYAN, C. (2005): "The ranking and rating of academics and journals in tourism research", Tourism Management, vol. 26, $\mathrm{n}^{\circ}$ 5, pp. 657-662.

SÁNCHEZ PÉREZ, M. y MARÍN CARRILLO, M.B. (2003): “La investigación en turismo y economía de la empresa publicada en revistas especializadas españolas: 1996-2001", Papers de Turisme, no 33, pp. 6-39.

SCIMAGO (2017): SJR - SCImago Journal \& Country Rank. Disponible en http://www. scimagojr.com.

VARGAS SÁNCHEZ, A. (2011a): “Los principales destinos son también las principales potencias en la investigación en turismo?”, Estudios turísticos, n 188, pp. 91-111.

VARGAS SÁNCHEZ, A. (2011b): "Tourism research in Spain: the state of the art", Enlightening Tourism, A Pathmaking Journal, n 1, pp. 93-110.

VERA, F. y IVARS, J. (2001): "La formación y la investigación turística en España: una visión de síntesis", Papers de Turisme, n 29, pp. 6-27.

ZHAO, W. y RITCHIE, J.R.B. (2007): “An investigation of academic leadership in tourism research: 1985-2004", Tourism Management, vol. 28, n 2, pp. 476-490.

ZHONG, L., WU, B. y MORRISON, A.M. (2015): "Research on China's tourism: A 35-year review and authorship analysis", International Journal of Tourism Research, vol. $17, \mathrm{n}^{\mathrm{o}} 1$, pp. 25-34. 
